\title{
Formulation Development of a Food-Graded Curcumin-Loaded Medium Chain Triglycerides-Encapsulated Kappa Carrageenan (CUR-MCT-KC) Gel Bead Based Oral Delivery Formulation
}

\author{
Kei-Xian Tan ${ }^{1,2, *}$, Ling-Ling Evelyn $\mathrm{Ng}^{1}$ and Say Chye Joachim Loo $1,3,4, *$ (D) \\ 1 School of Materials Science and Engineering, Nanyang Technological University, \\ Singapore 639798, Singapore; evelynisabellia@gmail.com \\ 2 Esco Aster, Block 71, Ayer Rajah Crescent, Singapore 139951, Singapore \\ 3 Singapore Centre for Environmental Life Sciences Engineering, Nanyang Technological University, \\ 60 Nanyang Drive, Singapore 637551, Singapore \\ 4 Harvard T.H. Chan School of Public Health, Harvard University, 677 Huntington Ave, \\ Boston, MA 02115, USA \\ * Correspondence: keixian.tan@escoaster.com (K.-X.T.); joachimloo@ntu.edu.sg (S.C.J.L.)
}

check for

updates

Citation: Tan, K.-X.; Ng, L.-L.E.; Loo, S.C.J. Formulation Development of a Food-Graded Curcumin-Loaded

Medium Chain Triglycerides-

Encapsulated Kappa Carrageenan (CUR-MCT-KC) Gel Bead Based Oral Delivery Formulation. Materials 2021, 14, 2783. https://doi.org/10.3390/ ma14112783

Academic Editor: Manuel Arruebo

Received: 19 April 2021

Accepted: 17 May 2021

Published: 24 May 2021

Publisher's Note: MDPI stays neutral with regard to jurisdictional claims in published maps and institutional affiliations.

Copyright: (c) 2021 by the authors Licensee MDPI, Basel, Switzerland. This article is an open access article distributed under the terms and conditions of the Creative Commons Attribution (CC BY) license (https:// creativecommons.org/licenses/by/ $4.0 /)$.

\begin{abstract}
In recent years, curcumin has been a major research endeavor in food and biopharmaceutical industries owing to its miscellaneous health benefits. There is an increasing amount of research ongoing in the development of an ideal curcumin delivery system to resolve its limitations and further enhance its solubility, bioavailability and bioactivity. The emergence of food-graded materials and natural polymers has elicited new research interests into enhanced pharmaceutical delivery due to their unique properties as delivery carriers. The current study is to develop a natural and food-graded drug carrier with food-derived MCT oil and a seaweed-extracted polymer called k-carrageenan for oral delivery of curcumin with improved solubility, high gastric resistance, and high encapsulation of curcumin. The application of k-carrageenan as a structuring agent that gelatinizes o/w emulsion is rarely reported and there is so far no MCT-KC system established for the delivery of hydrophobic/lipophilic molecules. This article reports the synthesis and a series of in vitro bio-physicochemical studies to examine the performance of CUR-MCT-KC as an oral delivery system. The solubility of CUR was increased significantly using MCT with a good encapsulation efficiency of $73.98 \pm 1.57 \%$ and a loading capacity of $1.32 \pm 0.03 \mathrm{mg}$ CUR/mL MCT. CUR was successfully loaded in MCT-KC, which was confirmed using FTIR and SEM with good storage and thermal stability. Dissolution study indicated that the solubility of CUR was enhanced two-fold using heated MCT oil as compared to naked or unformulated CUR. In vitro release study revealed that encapsulated CUR was protected from premature burst under simulated gastric environment and released drastically in simulated intestinal condition. The CUR release was active at intestinal $\mathrm{pH}$ with the cumulative release of $>90 \%$ CUR after $5 \mathrm{~h}$ incubation, which is the desired outcome for CUR absorption under human intestinal conditions. A similar release profile was also obtained when CUR was replaced with beta-carotene molecules. Hence, the reported findings demonstrate the potencies of MCT-KC as a promising delivery carrier for hydrophobic candidates such as CUR.
\end{abstract}

Keywords: drug delivery system; biodegradable; biomaterials; pharmaceutical; nutraceuticals

\section{Introduction}

Curcumin (CUR) is a biologically active, non-polar, and naturally occurring polyphenolic compound from turmeric that possesses a variety of health benefits [1]. It is the most active curcuminoid present in the flowering plant called Curcuma longa and is responsible for the yellow pigmentation of turmeric. Given its antimicrobial, antioxidant, antitumor, anti-inflammatory, and immunostimulatory properties, it has become one of the most widely studied food-derived compound [2]. Because of this, CUR is frequently used as a lipophilic compound in functional food. 
Despite its health benefits, its usage is limited by its poor water solubility, stability, and bioavailability $[3,4]$. CUR undergoes rapid degradation, molecular fragmentation, and metabolic inactivation at a physiological $\mathrm{pH}$. In addition, both alkaline and a neutral CUR solution can be degraded at room temperature and the degradation is more rapid at higher temperatures such as human body temperature $37^{\circ} \mathrm{C}$. CUR is also photochemicalsensitive whereby it undergoes degradation upon sunlight exposure. Because of its low water solubility $(\sim 11 \mathrm{ng} / \mathrm{mL})$, it usually ends up in feces [5]. Pharmacokinetic data shows that orally administered CUR in rodents and humans is low, including $0.22 \mu \mathrm{g} / \mathrm{mL}$ from $1 \mathrm{~g} / \mathrm{kg}$ CUR in mouse [6] and $0.051 \mu \mathrm{g} / \mathrm{mL}$ from $12 \mathrm{~g}$ CUR in humans [7]. To improve bioavailability, there is therefore a need to encapsulate CUR by enhancing its solubility.

One of the most suitable modes of delivery for CUR is through emulsions. Both CUR nano- and micro-emulsions have been extensively applied in drug delivery, the cosmetic sector, and food industry, for effective delivery of hydrophobic molecules [8]. However, the use of kappa ( $\mathrm{k}-)$ carrageenan $(\mathrm{KC})$ as a prime bulk phase structuring agent with gelation of CUR encapsulated in oil-in-water $(\mathrm{O} / \mathrm{W})$ emulsion is scarcely studied.

Medium-chain triglycerides (MCT) and KC employed in this study are generally recognized as safe (GRAS), non-toxic, edible, biodegradable, biocompatible, and biologically safe. These are interesting features for biopharmaceutical and food industries, owing to the contemporary direction of green consumerism. CUR in an edible medium such as MCT oil and KC, can be directly used in food or pharmaceutical ingredients, without the requirement of eliminating the extraction medium. MCT are triglycerides with two or three fatty acids and a short chain length of 6-12 carbon. It can be extracted from food sources such as coconut oil and palm kernel oil [9]. Moreover, the use of MCT in food products are approved with GRAS status by US FDA in 1994, which expands their applications in foods, cosmetics, nutrition, and drugs [10]. They are gaining increasing attention due to their unique bioactivities, such as lowering the cholesterol level, slowing weight gain, and increasing ketone production [11]. MCT as a carrier lipid has also been reported to significantly increase the CUR bioaccessibility $[9,12,13]$.

$\mathrm{KC}$, on the other hand, is a linear, anionic, hydrophilic, and sulphated polygalactan naturally derived from red seaweeds of the class Rhodophyceae [12]. It is made up of repeating D-galactose residues with one negative charge per disaccharide unit [13]. KC comprises D-galactose-4-sulfate and 3,6-anhydro-D-galactose. Due to its distinctive characteristics, carrageenan is extensively used in various biomedical applications and therapeutic treatments, as reported in numerous studies [14-17]. It has been exploited as the safe food additive for decades in meat, milk, and yogurt. This suggests the biocompatibility, functionality, and potencies of carrageenan as an ideal excipient for pharmaceutical delivery. Food-graded carrageenan has been deemed safe for its use in infant formulation (300 mg/L) by the Joint FAO/WHO Expert Committee on Food Additives (JECFA) [18] and it has been classified as non-carcinogenic by the International Agency for Research on Cancer (IARC) $[19,20]$. Furthermore, carrageenan is included in the British Pharmacopoeia 2012, US Pharmacopeia 35-National Formulary 30 S1 and European Pharmacopoeia 7.0, inferring its promising potential in the development of pharmaceutical formulations, taking into account its biological characteristics such as anti-herpes, anti-HIV, anticoagulant, anti-tumor, and immunomodulatory activities [21].

In the present study, a modified $\mathrm{O} / \mathrm{W}$ single emulsion technique is reported to prepare the CUR-MCT-KC with better properties by using MCT as a suitable oil phase for CUR encapsulation and $\mathrm{KC}$ as the aqueous phase that gelatinizes CUR-MCT emulsion into gel beads. Emulsion is a kinetically stable, non-equilibrated, and colloidal system comprising two or more immiscible liquids. There are various studies that have reported the use of either MCT or KC natural compounds for the encapsulation, delivery, and stabilization of CUR [1,9,22-26]. However, to the best of our knowledge, the use of both MCT oil and KC polysaccharides as an effective oral delivery carrier for CUR and their synergetic effects has never been reported before. 
This research work, the first of its kind, was able to fabricate CUR-MCT-KC gel beads and evaluate the efficiency of MCT oil and KC polysaccharides for CUR emulsification and high gastric resistance. The aim of this study was to design and develop a natural, food-graded oral delivery system for the delivery of CUR and examine the synergetic effects of MCT-KC for better encapsulation, solubility, and release of CUR. The in vitro experiments were carried out on the hypothesis that a MCT-KC nature-derived formulation can stabilize, encapsulate, and deliver CUR effectively via the gastrointestinal tract (GIT) when administrated orally. The differences between CUR-KC, CUR-MCT, and CURMCT-KC were also examined and compared to demonstrate the potential of CUR-MCT$\mathrm{KC}$ formulation. This work can therefore provide useful in vitro information for the development of an oral delivery system of other nutraceuticals, with poor solubility to further enhance their loading capacity and practical applications.

\section{Materials and Methods}

\subsection{Materials}

Curcumin (Curcuma longa (Turmeric), powder), KC (sulfated plant polysaccharide), phosphate-buffered saline (PBS) $(10 \times$ concentrate, $\mathrm{pH} 7.4)$, potassium chloride $(\mathrm{KCl})$ (molecular weight 74.55, $\geq 99 \%$ ), acetonitrile (ACN) (HPLC grade, $\geq 99 \%$ ), dichloromethane (DCM), bile salts (Dehydrated, purified fresh bile), Tween 20 (polysorbate 20), and Span 20 (sorbitan monolaurate) were purchased from Sigma Aldrich, Singapore. MCT oil (Neobee 1053) was purchased from Stepan company, Northfield, IL, USA. Deionized (DI) water was used in all the experiments.

\subsection{Preparation of Curcumin-Loaded Medium Chain Triglycerides-Encapsulated Kappa Carrageenan (CUR-MCT-KC) Bead Formulation}

CUR encapsulation was carried out using a single $\mathrm{O} / \mathrm{W}$ emulsion technique. This method is based on the emulsification of CUR-MCT organic solution into a water phase. Basically, the CUR molecules were first dissolved in MCT oil as an oil phase and Span 20 as the surfactant. The organic phase was then further emulsified in the continuous/aqueous phase made up of KC. Emulsification was conducted via homogenization under high-shear force to reduce the size of the CUR-MCT-KC emulsion droplet and thus, final particle size.

Initially, $5 \mathrm{mg}$ CUR was dissolved in $1 \mathrm{~mL}$ MCT oil and Span 20 (10\%) by stirring the mixture at $60{ }^{\circ} \mathrm{C}$ with gentle shaking at $150 \mathrm{rpm}$ for $5 \mathrm{~min}$ or until an orange solution was formed; $60{ }^{\circ} \mathrm{C}$ was adopted because it is a temperature that can be replicated in industrial conditions. The temperature was increased to $120{ }^{\circ} \mathrm{C}$ if the CUR was not dissolved completely; $2 \% w / v$ KC solution was prepared in distilled water by adding $2 \mathrm{~g}$ $\mathrm{KC}$ into $100 \mathrm{~mL}$ distilled water with gentle shaking at $350 \mathrm{rpm}, 60-70{ }^{\circ} \mathrm{C}$ for 5 min or until the complete dissolution of $\mathrm{KC}$ to obtain a clear and homogenous $\mathrm{KC}$ solution. To fabricate the $\mathrm{O} / \mathrm{W}$ emulsion, the KC solution was mixed with the MCT oils containing CUR $(0.5 \%$ $w / v$ CUR-MCT solution) via homogenization at $80{ }^{\circ} \mathrm{C}$ with $13,000 \mathrm{rpm}$ for $5 \mathrm{~min}$.

Finally, the CUR-MCT-KC bead production was conducted via a crosslinking process using $\mathrm{KCl}$ as crosslinkers. The above-prepared CUR-MCT-KC solution was dropped through a $1 \mathrm{~mL}$ syringe with a needle into a beaker containing a $5 \% \mathrm{KCl}$ aqueous phase. Beads were formed and collected in the aqueous phase, resulting in spherical CUR-MCTKC beads, as displayed in Appendix A (Figures A1 and A2). Next, the beads were collected and dried using filter papers prior to air-drying them in a light-protected container at room temperature for the beads to be hardened.

Similar procedures were carried out for the fabrication of CUR-MCT-KC beads containing a higher concentration of MCT (CUR-MCT-KC_2) using $5 \mathrm{~mL}$ MCT to dissolve $5 \mathrm{mg}$ CUR $(0.1 \%$ w $/ v$ CUR-MCT solution) before mixing with $0.4 \% w / v$ KC solution. On the other hand, beta-carotene-MCT-KC (BC-MCT-KC) beads were also fabricated using the same techniques and procedures by replacing CUR with $\mathrm{BC}$ molecules. 


\subsection{Biophysical Characterization of CUR-MCT-KC Formulation}

\subsubsection{Scanning Electron Microscopy (SEM)}

SEM JSM-6360 (JEOL, Ltd., Tokyo, Japan,) was used to visualize the size and surface morphology of the CUR-MCT-KC bead. SEM is a technology that uses the focused electron beams and different signals to obtain the sample image. The samples were mounted onto carbon tape prior to the sputter coating with gold for $45 \mathrm{~s}$. Each sample was then imaged at different magnification of $35 \times, 50 \times, 150 \times, 550 \times$, and $1000 \times$ with a $5 \mathrm{kV}$ voltage and $20 \mathrm{~mm}$ working distance.

\subsubsection{UV-Visible Spectrophotometric Analysis}

The presence of CUR can be identified by the maximum absorption peak that can be determined using UV-visible spectrophotometry at the wavelength of $425 \mathrm{~nm}$. This peak is mainly due to the $\pi-\pi$ type excitation of the CUR aromatic system [27]. The properties of the absorbance intensity changes at $425 \mathrm{~nm}$ were used to examine the solubility, dissolution, release, and stability of the fabricated CUR-MCT-KC formulation, using Infinite ${ }^{\circledR}$ M200 (Tecan Group Ltd, Menendorf, Switzerland). To ensure the measurement accuracy, $300 \mu \mathrm{L}$ volume was standardized for every analysis made. Moreover, the wavelength used for BC measurements was $448 \mathrm{~nm}$.

\subsubsection{Thermogravimetric Analysis (TGA)}

The thermal stability and behavior of CUR-MCT-KC beads was investigated using TGA (TA instrument Q500, TA Instruments, A Division of Waters Pacific Pte. Ltd., Singapore) to evaluate the change in mass at a constant heating rate in an inert environment. This is essential to determine the thermal stability and indicate the potential molecular rearrangement within the matrices of the CUR-MCT-KC complexation in terms of exotherm. The TGA instrument was purged with nitrogen at $20^{\circ} \mathrm{C} / \mathrm{min}$ and equilibrated at $25^{\circ} \mathrm{C}$ prior to the analysis. Approximately $13 \mathrm{mg}$ CUR-MCT-KC was placed in an aluminum pan and analyzed from 30 to $600{ }^{\circ} \mathrm{C}$ with $10^{\circ} \mathrm{C}$ per min of constant heating rate and $25 \mathrm{~mL} / \mathrm{min}$ of nitrogen gas. STARe software was applied for the result analysis.

\subsection{Chemical Analysis of CUR-MCT-KC Formulation}

In order to indicate the formation of CUR-MCT-KC and the presence of functional groups in CUR-MCT-KC, CUR, MCT oil, and CUR-MCT, prepared samples were characterized using the Fourier Transform Infrared Spectroscopy (FTIR) (Perkin Elmer Frontier, Waltham, MA, USA). Potassium bromide (KBr) was utilized as the background pellet and each sample (CUR, MCT, CUR-MCT, CUR-MCT-KC) was ground with the $\mathrm{KBr}$ in the ratio of 1:4 to form pellet using the hydraulic press. Spectra were measured in the range of $400-4000 \mathrm{~cm}^{-1}$ at a $4 \mathrm{~cm}^{-1}$ resolution. Sixteen scans for each sample were conducted to lower the sound-to-noise ratio.

\subsection{Determination of CUR Encapsulation Efficiency (EE)}

The quantity of CUR encapsulated within MCT-KC was evaluated from the difference between the initial amount of CUR added in the formulation and the amount of free CUR measured in the medium upon the breakdown of MCT-KC. Next, $10 \mathrm{mg}$ CUR-MCT-KC bead was added into $1 \mathrm{~mL}$ distilled water and heated at $60^{\circ} \mathrm{C}$ for $5 \mathrm{~min}$ or until a clear and homogenous solution was obtained and $1 \mathrm{~mL} A C N$ was added to the mixture prior to centrifugation at 13,000 rpm for $3 \mathrm{~min} ; 300 \mu \mathrm{L}$ supernatant was then collected for UVspectrophotometry analysis at $425 \mathrm{~nm}$. All absorbance readings were measured in triplicate and averaged. The CUR concentration was determined using the standard curve of CUR in ACN. The encapsulation efficiency (EE) was calculated from the following equation:

$$
\text { Encapsulation efficiency }(\%)=\frac{\text { Amount of encapsulated CUR }}{\text { Total initial amount of CUR added in CUR }-M C T-\text { KC beads }} \times 100 \%
$$




\subsection{Measurement of CUR Solubility in MCT Oil}

In $1 \mathrm{~mL}$ MCT oils, an excess amount of CUR, $10 \mathrm{mg}$ was added and heated to $60^{\circ} \mathrm{C}$ under stirring to ensure complete dissolution of CUR in MCT. The temperature was increased to $\sim 120{ }^{\circ} \mathrm{C}$ if the CUR was not dissolved completely. After cooling to $25^{\circ} \mathrm{C}$, the mixture was centrifuged at $13,000 \mathrm{rpm}$ for $5 \mathrm{~min}$ to collect the supernatant (CUR-saturated MCT oil) prior to the UV-spectrophotometry analysis at $425 \mathrm{~nm}$. The following equation was used to calculate the loading capacity:

$$
\text { Loading capacity }(\mathrm{mg} / \mathrm{mL})=\frac{\text { Amount of encapsulated CUR }}{\text { Total amount of MCT applied }}
$$

The Effect of Heat on the Solubility of CUR in MCT Oil

The effect of heat on the CUR solubility in MCT oil was studied over a temperature range of $37-100{ }^{\circ} \mathrm{C}$ using a water bath. $1 \mathrm{mg} / \mathrm{mL}$ CUR was prepared using MCT and heated from 37 to $100{ }^{\circ} \mathrm{C}$. At specific intervals $\left(37^{\circ} \mathrm{C}, 50{ }^{\circ} \mathrm{C}, 60^{\circ} \mathrm{C}, 70{ }^{\circ} \mathrm{C}, 80{ }^{\circ} \mathrm{C}, 90^{\circ} \mathrm{C}\right.$, $100{ }^{\circ} \mathrm{C}$ ), samples were collected to be examined spectrophotometrically at $425 \mathrm{~nm}$, as stated in Section 2.6 above. All absorbance readings were measured in triplicate and averaged.

\subsection{In Vitro Dissolution Study of CUR-MCT Formulation}

A dissolution study is essential to understand the solubility of naked/unformulated CUR as compared to a CUR-MCT mixture in PBS buffer that mimics the human physiological environment at $\mathrm{pH} 7.4$, temperature $37^{\circ} \mathrm{C}$, and $150 \mathrm{rpm}$ in a shaking incubator. Two different samples were prepared: (1) $4.15 \mu \mathrm{g} / \mathrm{mL}$ CUR solution prepared using PBS; the saturated CUR concentration reported in PBS; (2) $1.32 \pm 0.03 \mathrm{mg} / \mathrm{mg}$ CUR-MCT solution prepared using MCT; the saturated CUR concentration in MCT determined from our study. Samples were mixed with $5 \mathrm{~mL}$ PBS $(+0.02 \%$ Tween 20$)$ and incubated at $37^{\circ} \mathrm{C}, 150 \mathrm{rpm}$ for $1 \mathrm{~h}$. At every time interval (5th, 10th, 30th, 45th, 60th min), samples were collected for centrifugation and UV-spectrophotometer measurements at $425 \mathrm{~nm}$. The CUR concentration was determined using the standard curve of CUR in PBS. The percentage of CUR dissolved was calculated from the following equation:

$$
\text { Dissolved CUR }(\%)=\frac{\text { Dissolved CUR in PBS }}{\text { Total initial amount of CUR added }} \times 100 \%
$$

\subsection{In Vitro Release Study of CUR-MCT-KC Formulation}

\subsubsection{In Vitro Release Profile at Different $\mathrm{pH}$}

The effect of $\mathrm{pH}$ on encapsulation efficiency of CUR-MCT-KC and CUR-KC was evaluated and compared via the in vitro release study at extreme acidic and alkali $\mathrm{pHs:}$ $\mathrm{pH} 1.2$ and $\mathrm{pH}$ 7.4. $2 \mathrm{mg}$ of the CUR-MCT-KC bead was incubated in $1 \mathrm{~mL} \mathrm{PBS}(+0.02 \%$ Tween 20) at $\mathrm{pH} 1.2,150 \mathrm{rpm}$, and temperature $37^{\circ} \mathrm{C}$ for $2 \mathrm{~h}$ in a shaking incubator. At every time interval (5th, 15 th, 30th, 60th, 90th, 120 th $\mathrm{min}$ ), a supernatant was collected to be measured spectrophotometrically at $425 \mathrm{~nm}$ to identify the amount of released CUR. Fresh PBS was added to replenish the extracted sample at every time interval; the pH of PBS was adjusted using $0.1 \mathrm{M}$ sodium chloride $(\mathrm{NaCl})$ or $0.1 \mathrm{M}$ potassium hydroxide $(\mathrm{NaOH})$ to the respective $\mathrm{pH}$ level. The above procedures were repeated for CUR-MCT-KC bead under the alkali condition at $\mathrm{pH} 7.4$, and a release study of CUR-KC bead at both $\mathrm{pH} 1.2$ and $\mathrm{pH}$ 7.4. The percentage of CUR released was calculated from the following equation:

$$
\text { Released CUR }(\%)=\frac{\text { Released CUR from CUR }-M C T-K C \text { or CUR }- \text { KC beads }}{\text { Total initial amount of CUR added in CUR }-M C T-K C \text { or CUR }- \text { KC beads }} \times 100 \%
$$

\subsubsection{In Vitro Release Profile in Simulated GI Conditions}

The release mechanism of encapsulated CUR under simulated GI conditions was examined by mimicking the physiological environment in the upper tract (stomach and small intestine) of human GIT. The CUR-MCT-KC gel beads $(2 \mathrm{mg})$ were incubated in $1 \mathrm{~mL}$ 
simulated gastric fluid (SGF) $+0.02 \%$ Tween 20 at $\mathrm{pH} 1.2$, temperature $37^{\circ} \mathrm{C}, 150 \mathrm{rpm}$ in a shaking incubator for $2 \mathrm{~h}$, which represents the average transition time of GI. The entire sample after $2 \mathrm{~h}$ of gastric digestion was then transferred to the simulated intestinal fluid (SIF) $+0.3 \%$ bile salts for a subsequent incubation of $3 \mathrm{~h}$. At specific time intervals (5th, 15 th, 30th, 60th, 90th, 120th, 180th, 240th, 300th $\mathrm{min}), 1 \mathrm{~mL}$ supernatant was collected and mixed with $1 \mathrm{~mL}$ DCM prior to centrifugation at 13,000 rpm for $3 \mathrm{~min}$. This is to separate the released CUR from the loaded beads. Free CUR is very soluble in organic solvent-DCM. The CUR-DCM supernatant was collected and vacuum-dried. The released CUR was redissolved in $1 \mathrm{~mL} \mathrm{ACN}$ to assay spectrophotometrically at $425 \mathrm{~nm}$. All absorbance readings were measured in triplicate and averaged. Fresh SGF or SIF was added to replenish the extracted sample at every time interval. The concentration of released CUR was then determined using the standard curve of CUR in ACN. The percentage of CUR released was calculated from the following equation:

Released CUR $(\%)=\frac{\text { Released CUR from CUR }-M C T-K C \text { beads }}{\text { Total initial amount of CUR added in CUR }-M C T-K C \text { beads }} \times 100 \%$

The above procedures were replicated for in vitro release study of CUR-MCT-KC, CUR-MCT emulsion in simulated GI conditions, and BC-MCT-KC beads in different conditions: (1) SGF for $5 \mathrm{~h}$; (2) SIF for $5 \mathrm{~h}$; (3) SGF for the first $2 \mathrm{~h}$ and SGF for the subsequent $3 \mathrm{~h}$. Chloroform was used instead of DCM in the extraction of BC due to its solubility in different organic solvents. Moreover, the wavelength used for $\mathrm{BC}$ measurement was $448 \mathrm{~nm}$.

\subsection{Experimental Analysis}

Each block of experiment was conducted in triplicate (minimum), and the average measured values were reported as the final analytical data. Experimental data are recorded as average \pm standard deviation and/or standard error.

\section{Results and Discussion}

\subsection{Fabrication and Biophysical Characterization of CUR-MCT-KC Oral Delivery System 3.1.1. CUR-MCT-KC Design Concept and Optimization}

Based on the literature, the use of natural oils (e.g., corn oils, olive oils, black pepper oils, LCT oils, MCT oils) and natural polymers such as KC, as a delivery carrier for hydrophobic CUR molecules $[1,22,23,26,28-36]$, has been reported extensively. However, because of the gastric susceptibility of CUR, many reported formulations encounter the challenge of high gastric digestion. In this study, the aim was to report on a high encapsulation efficiency, a gastric-resistant oral delivery system for improved delivery of CUR. To the best of our knowledge, exploiting $\mathrm{KC}$ as a major bulk phase structuring agent with gelation of CUR encapsulated in $\mathrm{O} / \mathrm{W}$ emulsion is rarely reported. $\mathrm{KC}$ with strong gelling properties is capable of structuring and complexifying CUR-MCT into its helical form. Such a structure confers encapsulated CUR to be less susceptible to acid hydrolysis in GIT. It is hypothesized that random coiled KC chains are capable to interact with the CUR-MCT via hydrogen bonding between the KC polymeric chains and the glycerol molecules of MCT at elevated temperature. Upon cooling, $\mathrm{KC}$ undergoes gelation to rearrange into a more ordered, aggregated, and rod-shaped double helical conformation prior to the parallel aggregation of these double helices [37,38], which we hypothesize that this may strengthen the stability of CUR-MCT emulsion to form gel beads for GIT delivery. Meanwhile, there is so far no MCT-KC system established for the delivery of hydrophobic/lipophilic molecules. The aim of this work is also to fabricate MCT-KC gel beads to achieve a stronger gastric resistance, without the use of chemical solvents. The synthesized CUR-MCT-KC beads were solid and rigid with a spherical shape, regardless of the concentration of MCT used to dissolve the same amount of CUR molecules, as illustrated in Figure 1 below. However, CUR-MCT-KC_2 (with higher MCT concentration) beads were more yellowish in color, 
as seen in Figure $1 b$ ). This is due to a more even distribution and encapsulation of CUR within MCT oils with an increased volume.

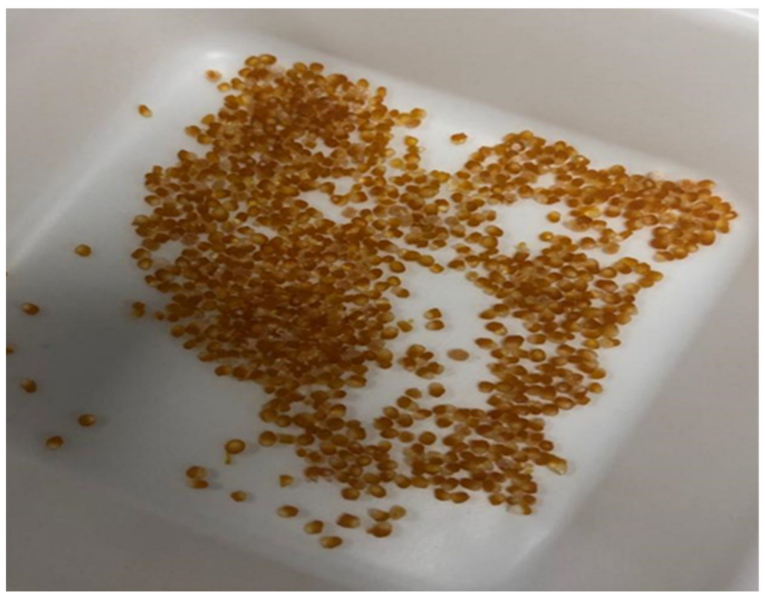

(a)

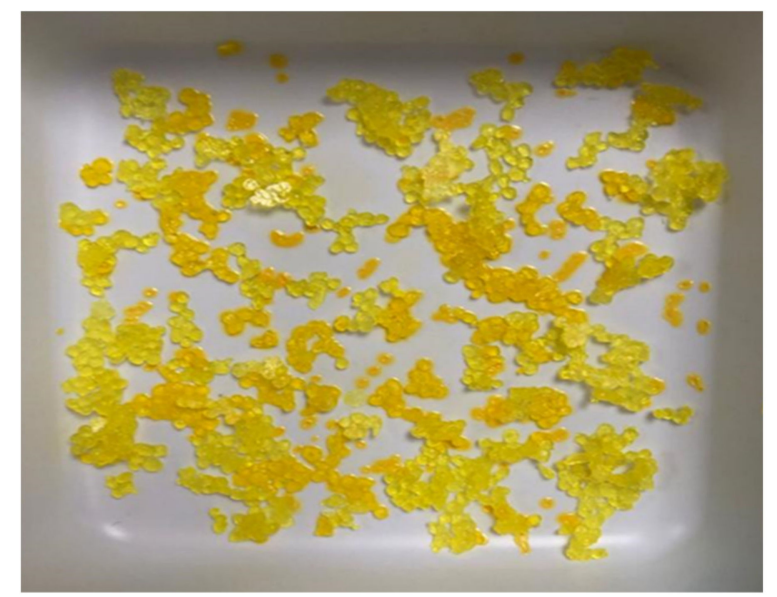

(b)

Figure 1. Dried and solidified CUR-MCT-KC and CUR-MCT-KC_2 beads with (a) $0.5 \% w / v$ CUR-MCT, (b) $0.1 \% w / v$ CUR-MCT before encapsulated with KC solution. Beads with higher amount of MCT were more yellowish due to a more even CUR distribution.

\subsubsection{Biophysical Characterization of CUR-MCT-KC Formulation SEM}

SEM was utilized to examine the size and surface morphology of the prepared CURMCT-KC formulation at different magnifications of $35 \times, 50 \times, 150 \times, 550 \times$, and $1000 \times$ in Figures 2 and 3. Figure 2 represents the SEM images of CUR-MCT-KC bead with a lower MCT concentration incorporated, whilst Figure 3 refers to CUR-MCT-KC_2 bead with a higher MCT concentration used to dissolve the same amount of CUR added. Particle shape, size, and surface chemistry play vital roles in the delivery and of encapsulated drugs. Both Figures 2 and 3 illustrate the bead-like morphology of CUR-MCT-KC. The bead was spherical in shape with a thicker coating, a rougher surface and lesser pores, suggesting the suitability of CUR-MCT-KC in achieving a more sustained drug release pattern [39]. A rough and less porous surface promotes better encapsulation capability, bead-cell interactions, and a lower release rate [40]. Furthermore, the rough surface of CUR-MCT-KC is advantageous to ease the effective surface modifications for enhanced cell targeting capability or a desired drug release profile.

CUR-MCT-KC_2 that was comprised of a higher amount of MCT was shown to be less spherical in shape with smoother and less porous surface features, as displayed in Figure 3. This indicates that the ratio of lipid and polysaccharide composition in a complex can significantly influence the morphological characteristics of the final product. The presence of more MCT oils contribute to a smoother bead surface and gel beads tend to be more spherical in shape with increasing amounts of polysaccharides, $\mathrm{KC}$ acting as the structuring agent. Nonetheless, the size of CUR-MCT-KC bead was recorded as $\sim 600 \mu \mathrm{m}$ regardless of the MCT concentration used to dissolve the same amount of CUR added initially. 

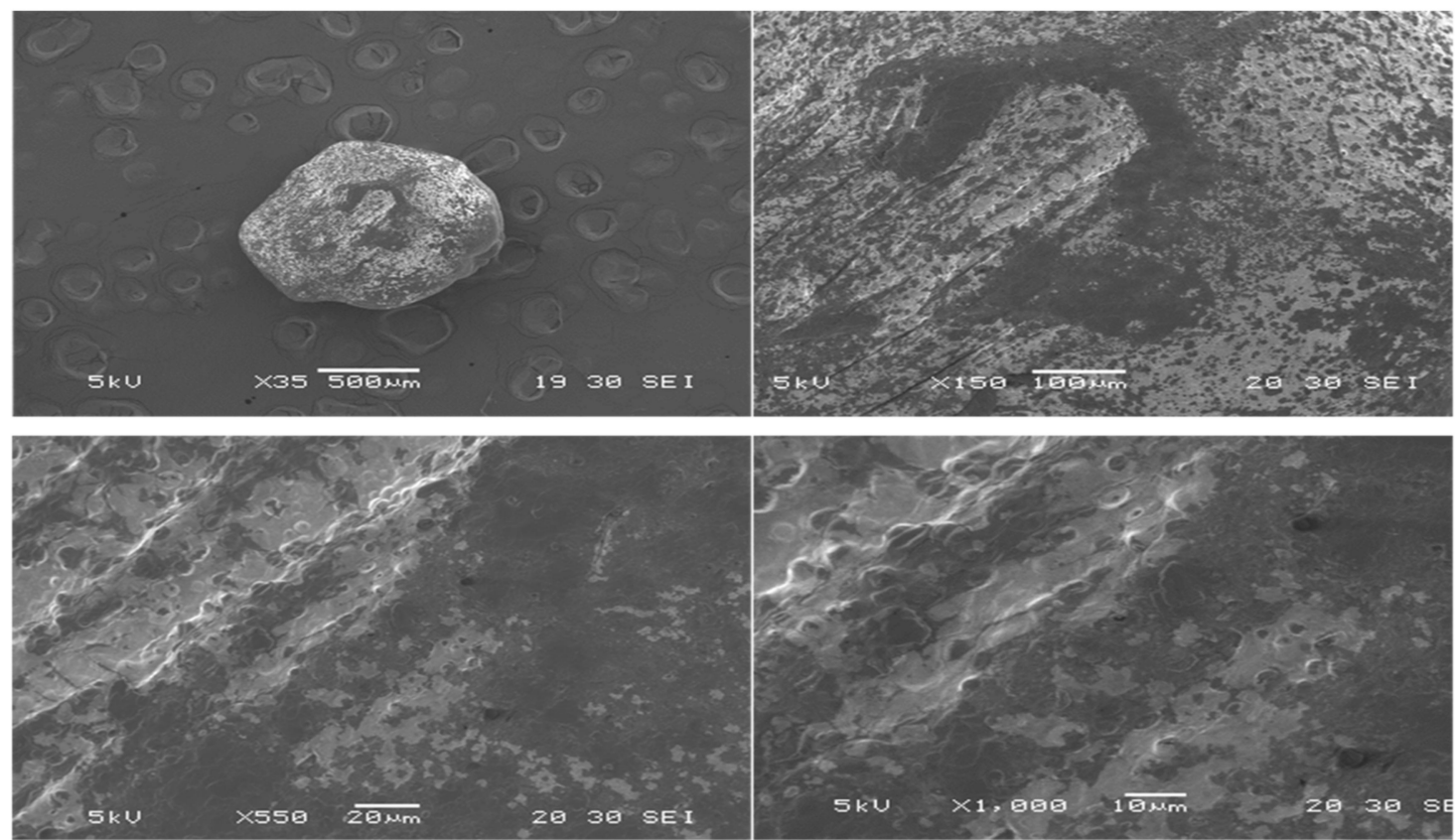

Figure 2. SEM analysis of CUR-MCT-KC bead showing spherical shape with moderately rough surface features. SEM analysis was performed at $5 \mathrm{kV}$ over a magnification of $\times 35$ to $\times 1000$.
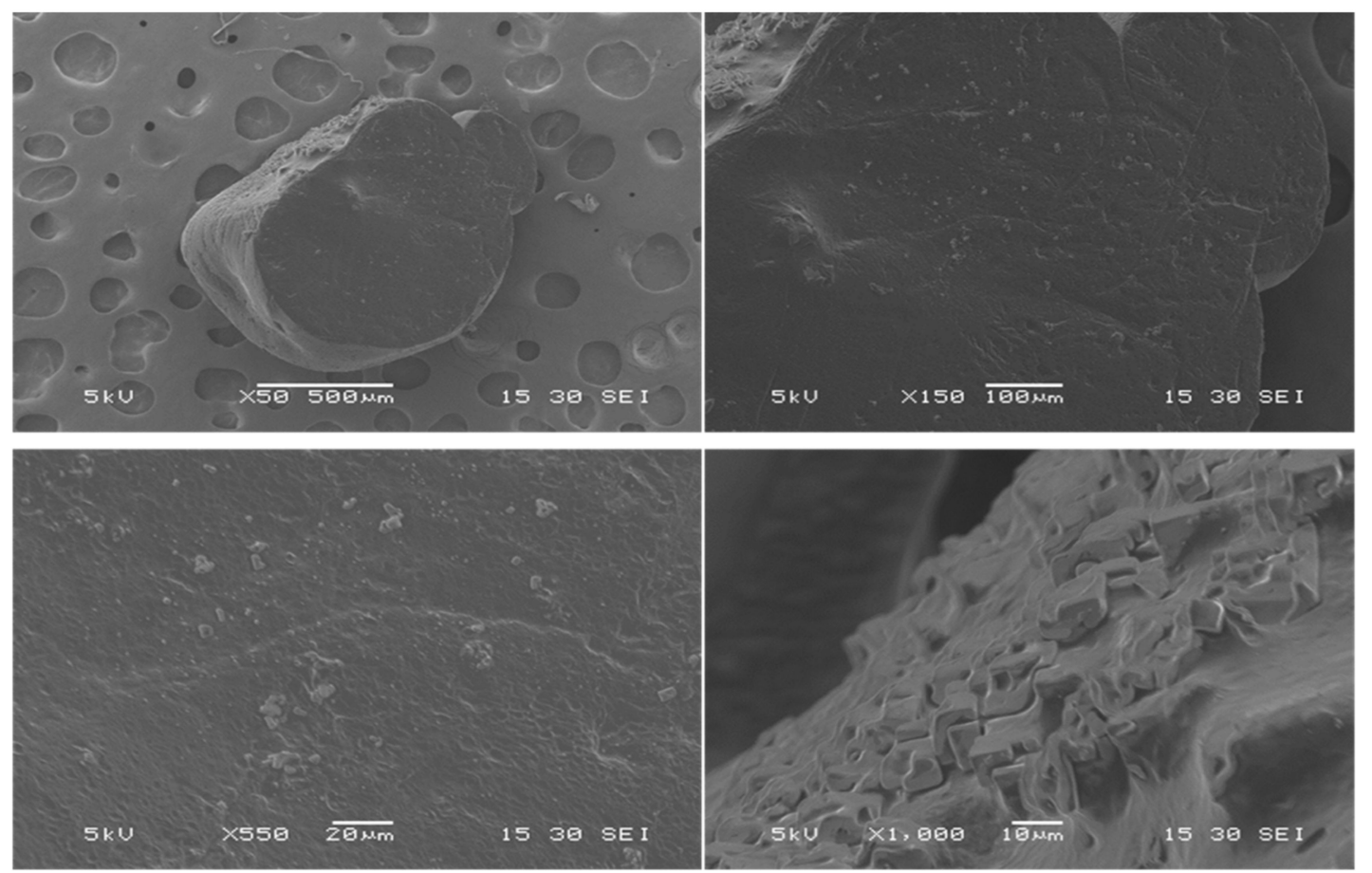

Figure 3. SEM analysis of CUR-MCT-KC_2 bead (higher MCT concentration used) showing smoother surface features. SEM analysis was performed at $5 \mathrm{kV}$ over a magnification of $\times 50$ to $\times 1000$.

TGA

The thermal behavior and stability of CUR-MCT-KC was studied using the TGA characterization. Figure 4 illustrates the TGA thermograms of CUR-MCT-KC. As seen in Figure 4, the primary heat-stimulated event was observed with a small slope between 150 and $200{ }^{\circ} \mathrm{C}$, due to a dehydration or desolvation process [41]. The maximum evaporation temperature was detected in the range of $30^{\circ} \mathrm{C}$ to approximately $200^{\circ} \mathrm{C}$. There was a sharp peak observed at approximately $320^{\circ} \mathrm{C}$ with $80.81 \%$ weight loss, which is related to the 
melting of CUR-MCT-KC, where it entered a melting phase and decomposition from 200 to $350^{\circ} \mathrm{C}$. CUR is in a crystalline form at an ambient temperature and the melting point of CUR was reported to be around $177^{\circ} \mathrm{C}$ [42]. This suggests that the encapsulation of CUR in MCT-KC helps to increase its thermal stability with a higher melting point. Degradation stopped at approximately $400{ }^{\circ} \mathrm{C}$, with a weight loss of $80.81 \%$ and a residue of $14.75 \%$. The TGA analytical result reveals CUR-MCT-KC with good thermal stability for its various applications in the industrial process and in storage.

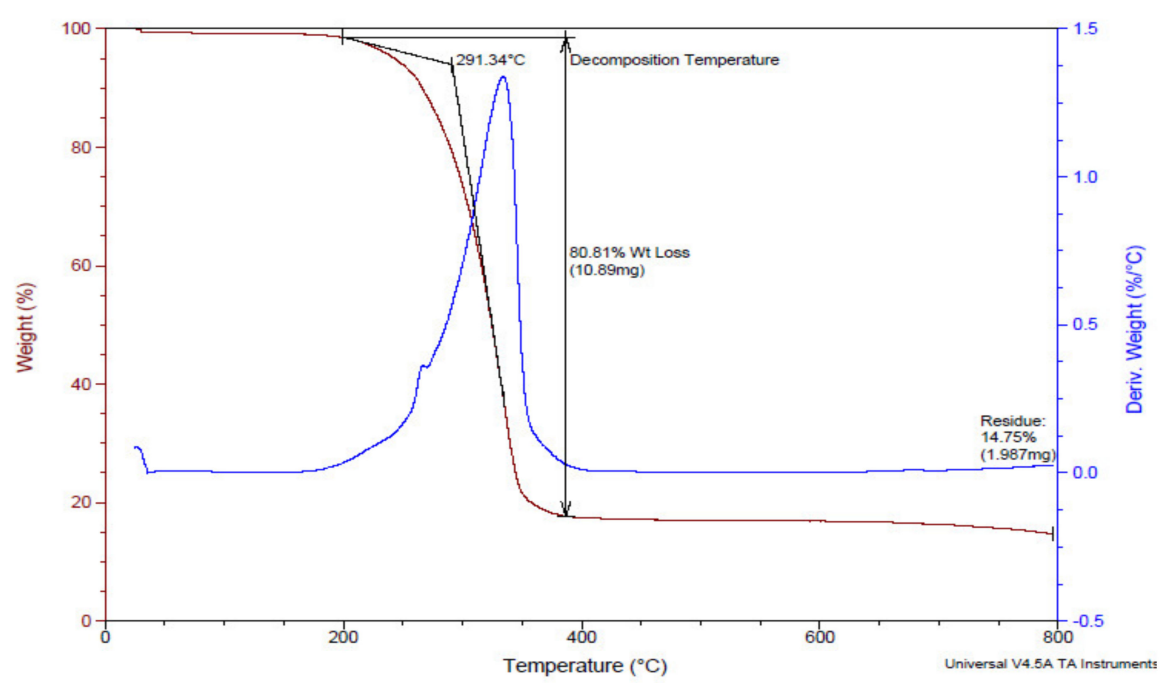

Figure 4. TGA analysis of CUR-MCT-KC bead to evaluate the change in weight at a constant heating rate of $10{ }^{\circ} \mathrm{C} / \mathrm{min}$ from 30 to $600{ }^{\circ} \mathrm{C}$. There was a sharp peak observed at approximately $320{ }^{\circ} \mathrm{C}$ with $80.81 \%$ weight loss, indicating the melting phase and decomposition of CUR-MCT-KC from 200 to $350{ }^{\circ} \mathrm{C}$.

FTIR

FTIR spectrum indices were used to differentiate between CUR, MCT, KC, and indicates the formation of CUR-MCT-KC formulation. The FTIR spectrum and vibrational characteristics of various functional groups presented in each component (CUR-MCT-KC, CUR-MCT, CUR, MCT) were identified as displayed in Figure 5 and were compared to other reported work. The FTIR bands and functional groups of pure CUR, MCT, and KC are shown in Appendix A (Table A1).

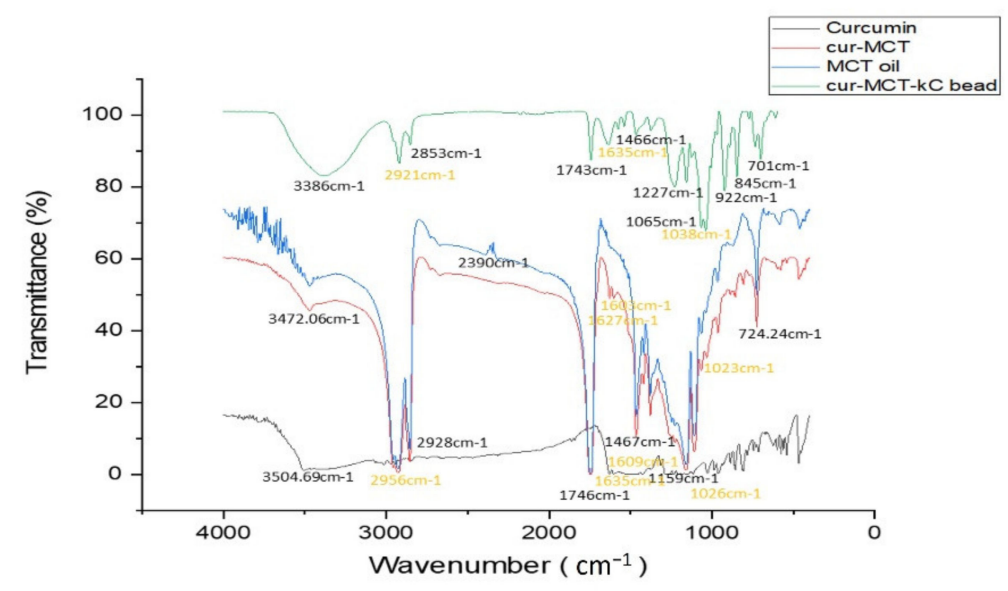

Figure 5. The FTIR spectra of the prepared CUR-MCT-KC, CUR, MCT oil, and KC in the range of $400-4000 \mathrm{~cm}^{-1}$ at a $4 \mathrm{~cm}^{-1}$ resolution. The yellow wavelengths indicated the successful CUR encapsulation whereby most of the active functional groups of CUR have been introduced in MCT as well as in the final formation of CUR-MCT-KC. 
Regarding the solubility and encapsulation of CUR in MCT to form CUR-MCT, the FTIR spectra indicates most of the active functional groups of CUR have been introduced in MCT. This includes $3504 \mathrm{~cm}^{-1}, 1603 \mathrm{~cm}^{-1}, 1627 \mathrm{~cm}^{-1}, 1023 \mathrm{~cm}^{-1}$, and $724 \mathrm{~cm}^{-1}$ attributed to the phenolic $(\mathrm{OH})$, carbonyl $\mathrm{C}=\mathrm{O}$ and alkenes $\mathrm{C}=\mathrm{C}$, benzene ring, $\mathrm{C}-\mathrm{O}-\mathrm{C}$ stretching vibrations, and $\mathrm{CH}_{2}$ stretching vibrations of $\mathrm{CUR}$, respectively. On the other hand, the chemical interactions and the formation of CUR-MCT-KC were confirmed, as shown in Figure 5 with the presence of major peaks of CUR and MCT in KC such as the $3386 \mathrm{~cm}^{-1}$ peak with slight spectral changes due to interactions of CUR and MCT. On top of that, other functional groups associated with $1635 \mathrm{~cm}^{-1}, 1605 \mathrm{~cm}^{-1}$, and $1038 \mathrm{~cm}^{-1}$ peaks showed the encapsulation of CUR; $2923 \mathrm{~cm}^{-1}, 1743 \mathrm{~cm}^{-1}$, and $1466 \mathrm{~cm}^{-1}$ identified the incorporation of MCT, and $922 \mathrm{~cm}^{-1}, 845 \mathrm{~cm}^{-1}$, and $701 \mathrm{~cm}^{-1}$ revealed the presence of KC. Hence, the presence of CUR, MCT, and KC functional peaks in the FTIR analysis indicates the conjugated composite materials of CUR-MCT-KC beads.

\subsubsection{Encapsulation Efficiency (EE) of CUR-MCT-KC Formulation}

EE is the percentage of CUR being entrapped successfully into the MCT-KC formulation. The CUR-MCT-KC_2 bead exhibited a significantly higher EE (73.98 $\pm 1.57 \%)$ than CUR-MCT-KC bead $(\sim 24.04 \pm 2.17 \%)$. EE is therefore relatively dependent on the amount of MCT oil used. This also indicates that the use of MCT can improve the solubility of CUR to a greater extent. Their storage stability was also examined with CUR-MCT-KC_2 beads showing a good stability of up to at least 15 days at room temperature (EE of $69 \pm 0.034 \%$ ), and 30 days of storage at $-20{ }^{\circ} \mathrm{C}(\mathrm{EE}$ of $71 \pm 0.018 \%)$. Hence, CUR-MCT-KC_2 beads were selected for further investigations in terms of solubility, dissolution, and drug release studies.

The surface charge and structural diversity of the encapsulation materials used can notably affect their efficiency in encapsulating and retaining CUR. Initially, CUR interacted with the fatty acid chains of MCT that aided in its solubilization. Ma, Zeng [43] suggested that MCT improves the dipole-dipole interactions between its polar groups and CUR molecules, resulting in an enhanced CUR solubility. Furthermore, MCT consists of oxygen molecules that allow the formation of hydrogen bonds with CUR on top of their effective dipole-dipole interactions [31]. Therefore, with an increase in MCT concentration, there are more dipole-dipole and hydrogen bonds formed, leading to better CUR loading and EE. The solubility of CUR in MCT is significantly higher than in other common oils. This is due to MCT oil, which possesses shorter acyl chains (C6-C10) and higher polarity as compared to other common oils (C16-C20) such as corn, soybean, olive and rapeseed oils, and LCT oils. Hence, the polarity of MCT is more suitable for interactions with CUR molecules [25], demonstrating its effectiveness as a CUR delivery carrier.

Besides this, the emulsifier plays an important role in the formation of a stable emulsion. Span 20 with an intermediate hydrophilic-lipophilic balance value of 7-9 was used in this study. It was determined to improve the CUR solubility and effectively prevent CUR crystallization, even after being stored at room temperature for months [24]. The presence of Span 20 had lowered the interfacial tension, resulting in better CUR solubility in MCT. KC was used to encapsulate those loosely surface bound CURs and act as a protective layer on top of the MCT compartment against harsh conditions such as the acidic environment of the stomach.

The EE $(73.98 \pm 1.57 \%)$ of CUR-MCT-KC_2 bead is highly comparable and performs better than other reported CUR-loaded formulations including CUR-MCT nanoemulsion with $71.5 \%$ EE [24], CUR-KC complex with 73.6\% EE [22], CUR-MCT organogel with $\sim 2.6 \%$ EE [9], CUR organogel-based nanoemulsion with 9\% EE [34], CUR-encapsulated caseinate/zein nanoparticles with 62\% EE [44], CUR-KC film for food freshness monitoring with 3\% EE [28], and CUR-KC drug carrier to treat A549 lung cancer cells with 73\% EE [22]. It is important to note that most of the reported CUR-MCT nano/micro emulsions focused on improving the lipolysis and bioaccessibility of CUR. Hence, unlike our present work, the efficiency of CUR encapsulation and loading in MCT were not studied extensively in 
most cases. In short, CUR-MCT-KC_2 formulation possesses better encapsulation efficiency than other existing CUR carriers, confirming its suitability to carry the hydrophobic CUR with increased solubility and stability.

\subsection{Interactions of Hydrophobic CUR with MCT Oil \\ 3.2.1. Solubility of CUR in MCT Oil}

The solubility of CUR in MCT oil was examined in this study to understand the loading capacity of CUR per $\mathrm{ml}$ of MCT used. The result reveals that the solubility of CUR in MCT was $1.32 \pm 0.03 \mathrm{mg} / \mathrm{mL}$, which is more than 100 times higher than the solubility of unformulated CUR in PBS $(4.15 \mu \mathrm{g} / \mathrm{mL})$ [45]. This can be ascribed to the shorter acyl chains and the presence of a higher number of polar groups (oxygen) of MCT that enhance the dipole-dipole interactions between CUR molecules and MCT. The obtained result is also the same or better than other reported CUR-MCT formulations, including CUR nano-emulsion with a solubility of $0.25 \mathrm{mg} / \mathrm{mL}$ [23], $1.85 \mathrm{mg} / \mathrm{g}$ [25], $0.79 \pm 0.2 \mathrm{wt} \%$ [33], and $2.9 \mathrm{mg} / \mathrm{g}$ [32] in MCT oil. This validates the efficiency of MCT as an oil carrier for CUR encapsulation.

\subsubsection{The Effect of Heat on the Solubility of CUR in MCT}

CUR molecules were readily dispersed in MCT oil with increased heat, as seen in Appendix A (Figure A3). The formation and stability of CUR-MCT was investigated based on the maximum absorption peak at $425 \mathrm{~nm}$ using UV-visible spectrophotometry. The prepared CUR-MCT emulsion exhibited a shoulder peak at $425 \mathrm{~nm}$ due to the $\pi-\pi$ type excitation of the CUR aromatic system, indicating the presence and stability of CUR in MCT oil. Changes in the absorbance intensity at $425 \mathrm{~nm}$ were then applied to evaluate the stability of CUR-MCT emulsion under the effect of heat when the temperature increased substantially over time. Figure 6 illustrates that the absorbance intensity increased gradually over the incubation period with increasing temperature, revealing that the solubility of CUR in MCT was enhanced under the influence of heat. Hence, CUR was able to be dissolved in heated MCT oil without degrading and remained soluble for a relatively long period of time with a stable CUR-MCT-KC bead formed, demonstrating good stability. This was proven with the $71 \%$ EE of stored CUR-MCT-KC bead at $-20{ }^{\circ} \mathrm{C}$ after one month of storage time, which was close to the $\mathrm{EE}(73.98 \pm 1.57 \%)$ measured right after the fabrication process. This result is aligned with other research studies, which applied heat to improve the encapsulation of CUR in MCT $[9,26,33,34]$. CUR formulations would have a higher retention rate and stability if they were stored at lower temperatures, in order to prevent degradation by Ostwald ripening [46]. In addition, many reported studies exemplified the high stability of CUR in MCT after a 30-day storage period at different temperatures, including room temperature at $4{ }^{\circ} \mathrm{C}$ and $-20{ }^{\circ} \mathrm{C}$ [23]. Therefore, the application of heat is suggested to improve the CUR solubility and stability for an efficient CUR encapsulation.

\subsubsection{In Vitro Dissolution Study of CUR-MCT Formulation}

CUR is not soluble in the water phase and its solubility is extremely low, even with the application of emulsifiers. Dissolution is defined as the rate of solute dissolving in a solution where it is a kinetic process and is measured by its rate. A simple in vitro experiment was carried out to determine the amount of CUR dissolved in PBS after an incubation period of $1 \mathrm{~h}$, with/without the presence of MCT. PBS was used in the study to mimic the human physiological environment. Unlike the unformulated or naked CUR, MCT-solubilized CUR was shown with a greater in vitro dissolution rate in PBS. The data, as seen in Figure 7, suggests that CUR-MCT has a higher dissolution rate as compared to unformulated CUR alone when incubated in PBS (pH 7.4) at $37^{\circ} \mathrm{C}, 150 \mathrm{rpm}$ for an hour. The results determined that approximately 33\% unformulated CUR and $\sim 67 \%$ CUR-MCT were dissolved in PBS, respectively. 


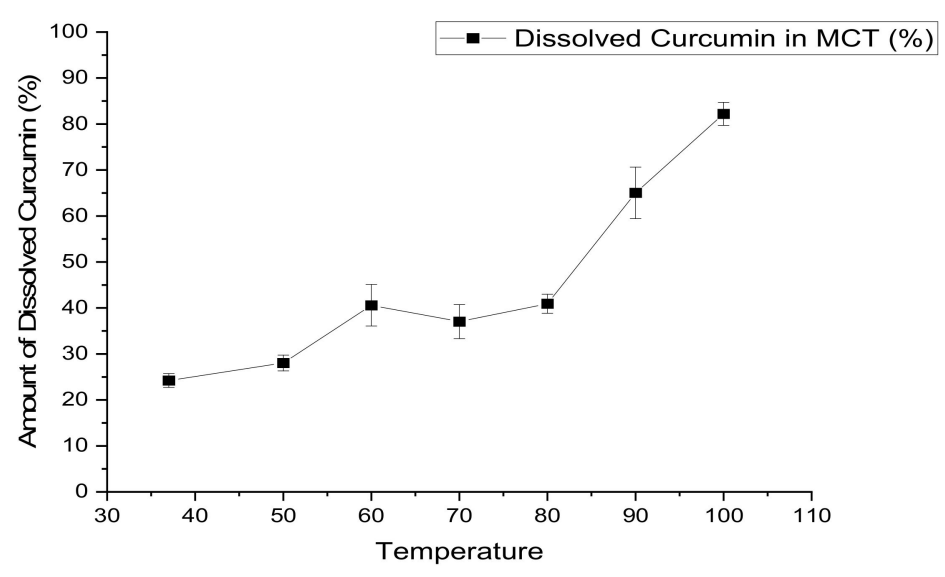

Figure 6. The amount of dissolved CUR (\%) in heated MCT oil over a range of temperature from 37 to $100{ }^{\circ} \mathrm{C}$ in a water bath. The application of heat is suggested to improve the CUR solubility and stability in encapsulation.

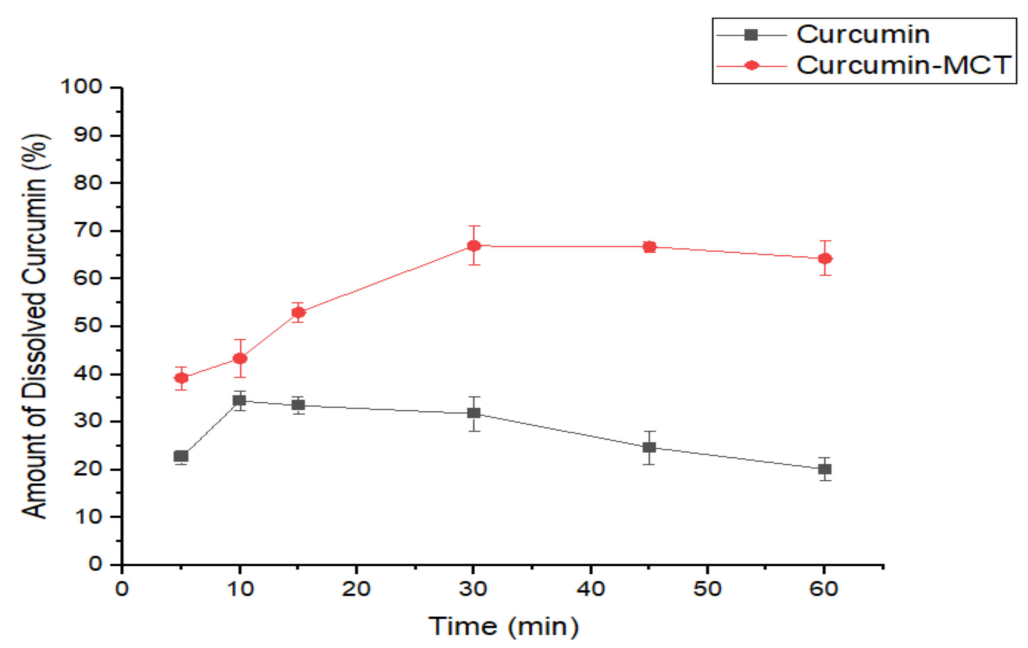

Figure 7. The in vitro dissolution test of unformulated CUR and CUR-MCT was conducted in PBS over $60 \mathrm{~min}$ at temperature $37^{\circ} \mathrm{C}$ with gentle shaking of $150 \mathrm{rpm}$ in a shaking incubator. MCT plays an important role in enhancing the CUR solubility.

The unformulated CUR in PBS started to degrade gradually and only around $22 \%$ was detected after $1 \mathrm{~h}$ of incubation as compared to its initial amount added. The poor solubility of unformulated CUR $(\sim 33 \%)$ is in keeping with other studies, which revealed the high hydrophobicity and poor solubility of unformulated CUR $[47,48]$. This is due to the low water solubility of $<0.005 \mathrm{wt} . \%$ and the high oil-water partition coefficient $(\log \mathrm{P} 3.1)$ of CUR. There are also studies [49-51] that have demonstrated that most of the CUR (more than $90 \%$ ) is degraded rapidly within 30 min of incubation in PBS and this is similar to our findings, as shown in Figure 7, that the amount of solubilized CUR decreased drastically after 15 min in PBS ( pH 7.4). In contrast, the stability of CUR increased when it was loaded in MCT, whereby CUR encapsulation inside the oil globule helps to minimize the contact of CUR with the external PBS environment. Figure 7 reflects that the presence of MCT can enhance the solubility of CUR in PBS, at which $\sim 65 \%$ CUR-MCT was still detectable after an hour of incubation, suggesting that the solubility of CUR-MCT in PBS was increased two-fold. This indicates the significant role of MCT compartment in MCT-KC gel beads for the encapsulation of hydrophobic/lipophilic molecules. 


\subsection{In Vitro Release Study of CUR-MCT-KC Formulation in Simulated GI Conditions}

The digestion of delivery carrier in the GIT is a complex mechanism and its impact on the release of encapsulated bioactive compounds plays an important role in the uptake, distribution, and bioavailability of the encapsulated compound. Both MCT and KC were evaluated for their entrapment efficiency for CUR via the in vitro release study under human physiological conditions. This study is important to demonstrate the synergetic effects of both MCT and KC as an oral delivery carrier. The conditions of an incubating medium could crucially affect the drug release profile. Hence, both the gastric and intestinal digest were investigated in vitro for the cumulative release of CUR from MCT-KC beads, as seen in Figures 8 and A4 (Appendix A).

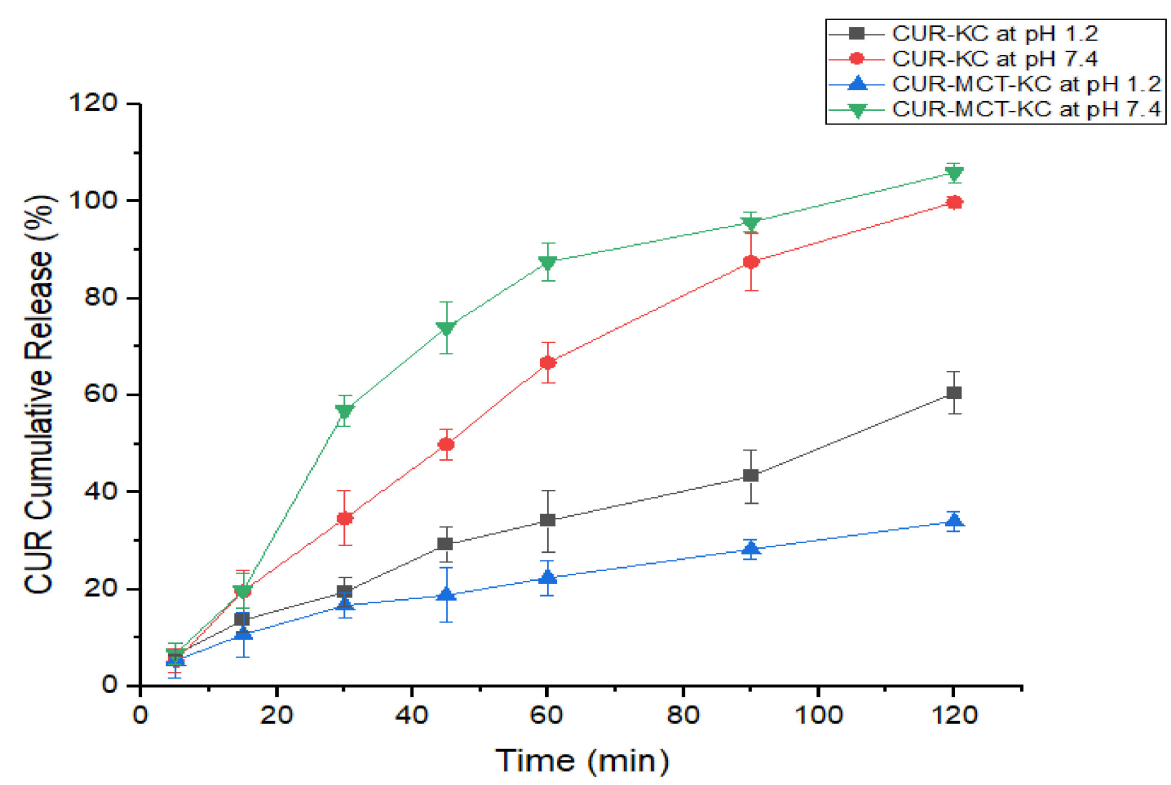

Figure 8. Cumulative release characteristics of CUR from CUR-KC and CUR-MCT-KC under the influence of different $\mathrm{pH}$ : acidic $\mathrm{pH} 1.2$ and alkali $\mathrm{pH}$ 7.4. The release experiment was the performance at $37^{\circ} \mathrm{C}$ in a PBS buffering system, with gentle shaking at $150 \mathrm{rpm}$ over $2 \mathrm{hr}$. CUR release (\%) was monitored at specific intervals: 5th, 15th, 30th, 45th, 60th, 90th, 120th min.

\subsubsection{CUR-KC vs. CUR-MCT-KC at Different $\mathrm{pH}$}

The optimal hydrolysis of $\mathrm{KC}$ is largely dependent on the $\mathrm{pH}$ of the reaction mixture. As displayed in Figure 8, the CUR release rate was higher at $\mathrm{pH} 7.4$ in the intestinal environment for both CUR-KC and CUR-MCT-KC, than at $\mathrm{pH} 1.2$ in the gastric condition. This can be explained due to $\mathrm{KC}$ hydrolysis, which is optimum at a varying $\mathrm{pH}$ above $\mathrm{pH} 7$, such as $\mathrm{pH} 7.5$ [52] and $\mathrm{pH} 7.7$ [53], and in the order of $\mathrm{pH} 7.4>0.1 \mathrm{M} \mathrm{HCl}>$ distilled water [54]. These are consistent with our findings that the $\mathrm{KC}$ degradation rate was higher at a neutral $\mathrm{pH}(\sim \mathrm{pH} 7.4)$ with a rapid release of CUR molecules under the intestinal environment.

In this study, $\mathrm{KCl}$ was demonstrated as an effective crosslinker whereby $\mathrm{KC}$ became more resistant to acid hydrolysis in the stomach upon the crosslinking with $\mathrm{K}^{+}$ions [55]. The presence of certain cations such as sodium $\left(\mathrm{Na}^{+}\right)$, calcium $\left(\mathrm{Ca}^{+}\right)$, or $\mathrm{K}^{+}$ions strongly enhances the stability of the ordered helices and aggregations, which further promotes gelation, resulting in a slower depolymerization rate of $\mathrm{KC}$ in acidic condition. This describes the effectiveness of fabricating MCT-KC via a $\mathrm{KCl}$ crosslinking process in this study to obtain a more solidified carrier for hydrophobic molecules with better protective outcomes. Based on Figures 8 and A4 (Appendix A), the suitability of KC in oral drug delivery was exemplified with its capability to prevent premature release and degradation of an encapsulated drug [22]. There was no initial burst at the first $15 \mathrm{~min}$ and less than $35 \%$ CUR was released after $1 \mathrm{~h}$ gastric incubation for both CUR-KC and CUR-MCT-KC 
beads. This elucidates the selection of $\mathrm{KC}$ as one of the carrier materials in this work. Importantly, CUR-MCT-KC exhibited a much lower CUR release $(<30 \%)$ at the end of $2 \mathrm{~h}$ gastric incubation as compared to CUR-KC, suggesting that the presence of MCT further improves the acid resistance.

\subsubsection{In Vitro Release Profile of CUR-MCT-KC}

In SGF

In the initial phase, the release (\%) of CUR from CUR-MCT-KC was gradual and there was no initial burst observed at the first hour of incubation in SGF at $37^{\circ} \mathrm{C}$ for $2 \mathrm{~h}$, indicating that CUR-MCT-KC was relatively resistant to pepsin digestion. This ensures that the encapsulated CUR is delivered and released in the targeted intestinal region. When incubated in SIF for the following $3 \mathrm{~h}$, CUR-MCT-KC was capable of releasing the maximum amount ( $>90 \%$ ) of entrapped CUR between the third and the fourth hour of incubation in SIF, as seen in Figures 9 and 10. This illustrates an efficient release of CUR under mimicked intestinal conditions, allowing further CUR absorption into the human circulatory system. A delivery system with an efficient release at the targeted site is essential for CUR to be able to elicit its bioactivity.

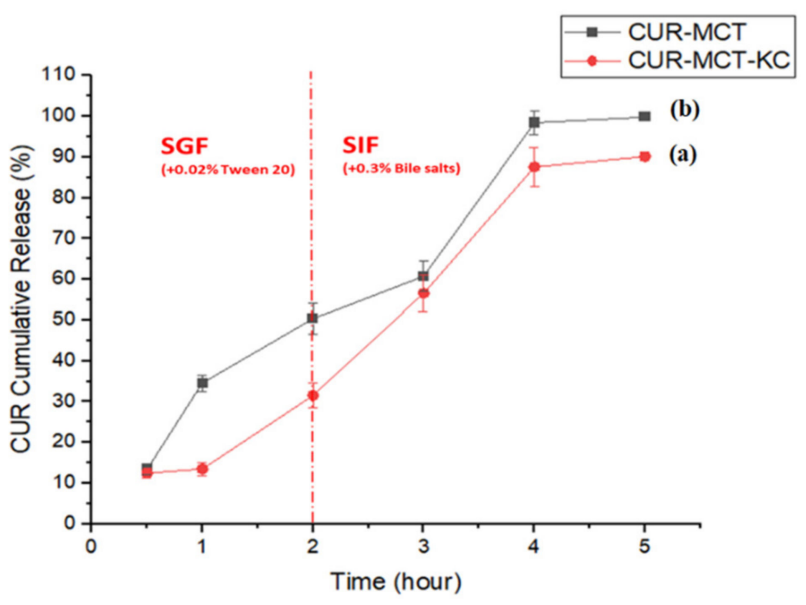

Figure 9. Cumulative in vitro drug release profile of CUR from (a) CUR-loaded-MCT-KC beads and (b) CUR-MCT emulsions at $37^{\circ} \mathrm{C}$ with gentle shaking, $150 \mathrm{rpm}$ in SGF (pH 1.2) for $2 \mathrm{~h}$ incubation and followed by the subsequent $3 \mathrm{~h}$ incubation in SIF ( $\mathrm{pH}$ 6.8).

The release pattern of CUR-MCT-KC has specified its suitability for oral administration of drugs. Oral delivery is one of the most preferred routes, due to its high patient compliance. As shown in Figure 9, the slow release of CUR in the first $2 \mathrm{~h}$ has proved the resistance of MCT-KC against the harsh gastrointestinal environment of the stomach in order to avoid burst release and elicit better shielding effects for the encapsulant in the localized environment. Moreover, carrageenan could maintain its ionization at a low $\mathrm{pH}$ owing to their low $\mathrm{pKa}$ value, suggesting their potencies as gastric floating tablets [56]. In this study, the addition of monovalent cations such as $\mathrm{KCl}$ has resulted in a stronger $\mathrm{KC}$ gel [57] due to the ion pairs formed between $\mathrm{KC}$ and added $\mathrm{KCl}$ crosslinker. As a result, CUR-MCT-encapsulated KC forms a mesh or network-like layout to enhance the CUR retention and protective effects to a certain degree. This is an important feature of $\mathrm{KC}$ in providing an effective encapsulation when KC helices interact with CUR-MCT molecules to form a complex structure, leading to a higher solubility. The solubility can be enhanced by 15 to 30 times as compared to the free compounds due to their conformational changes into an amorphous setting in the complex structure [58]. On top of that, $\mathrm{KC}$ is employed in this work as it is often used as an excipient for bead fabrication due to its viscoelastic, easy gelling, and thermo reversible properties for a prolonged retention and controlled drug release [59]. In short, MCT-KC may act as a potential carrier to deliver and release hydrophobic/lipophilic drug molecules, specifically at intestinal regions. 


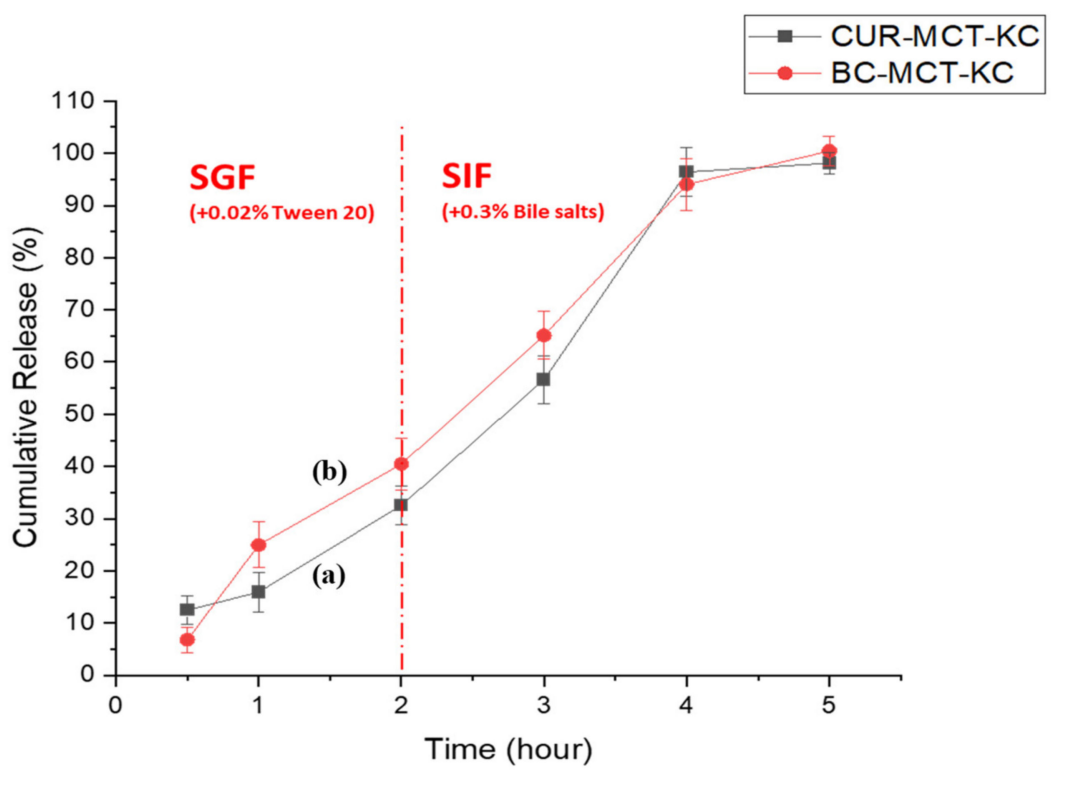

Figure 10. The in vitro release kinetics of MCT-KC bead in carrying two different types of hydrophobic molecules: (a) CUR, (b) BC. The cumulative release rate was investigated in simulated gastric $\left(\mathrm{pH}\right.$ 1.2) and intestinal digestion $\left(\mathrm{pH}\right.$ 6.8) for a $5 \mathrm{~h}$ incubation period, at $37^{\circ} \mathrm{C}$ with gentle shaking at $150 \mathrm{rpm}$. Both curcumin-MCT-kC and beta carotene-MCT-kC beads showed similar release kinetics. There was no initial burst at the first hour and a significant release was observed at the second $(<40 \%$ cumulative release) hour and an approximately $100 \%$ cumulative release was achieved at the fourth hour of incubation. Data are expressed as the mean \pm standard error of three independent experiments.

\section{CUR-MCT Emulsion vs. CUR-MCT-KC Beads}

The release profile of the CUR-MCT-KC bead was compared to that of the CUR-MCT emulsion, as illustrated in Figure 9. The CUR release of the CUR-MCT emulsion was more than $50 \%$ after the $2 \mathrm{~h}$ gastric incubation as compared to CUR-MCT-KC beads with $\sim 30 \%$ CUR release under the simulated gastric condition. This validated the efficiency of $\mathrm{KC}$ as an effective structuring agent for the encapsulation and gelation of CUR-MCT emulsions. The release profile of the CUR-MCT emulsion and the CUR-MCT-KC bead are useful as references to investigate the release pattern of MCT encapsulation, which is scarcely reported. Most reported works [1,9,23,32-35] focus on the bio-accessibility and lipolysis of MCT encapsulation in the intestinal region. However, these reported studies do not provide an in-depth understanding regarding the effectiveness of its GIT delivery in terms of encapsulation efficiency and gastric resistance of MCT as a drug carrier towards intestinal absorption. Some studies have supported the efficiencies of $\mathrm{KC}$ encapsulation, which includes the use of KC in delivering probiotic bacteria such as Lactobacillus plantarum and Lactobacillus rhamnosus, as well as other poorly soluble drugs for enhanced GIT delivery [22,60-62]. The release of CUR in SGF can be attributed to the normal swelling and hydrolysis of $\mathrm{KC}$ and thus contributed to the increasing release of CUR detected at the 2nd h of SGF incubation. This outcome is highly desired, because the degradation of KC can further expose the CUR-MCT compartment to SIF to ease the digestion of MCT and increase the release of CUR within the intestinal lumen.

\section{Stomach $\mathrm{pH}$ of Fasted- and Fed-Stated}

In this study, the $\mathrm{pH}$ used to mimic the gastrointestinal condition was extreme, at around $\mathrm{pH}$ 1.2. This was to investigate the protective effect of $\mathrm{KC}$ under the most unfavorable and rapid acidification of gastric digestion. In fact, under the in vivo condition or the human physiological environment, the gastric of GIT is acidified gradually, whilst gastric emptying is initiated as soon as food ingestion takes place. The gastric $\mathrm{pH}$ of a fasted-and 
fed-stated is $\sim \mathrm{pH} 1.3$ and $\mathrm{pH} 5$, respectively in healthy subjects due to the buffering effects of ingested meals $[63,64]$. Hence, the result recommends that KC can even offer a better protective effect against the acidic $\mathrm{pH}$ during the fed-state and gastric emptying process.

In SIF

Pancreatic enzymes, also known as pancreatin, are commercial mixtures of lipase, protease, and amylase enzymes [65]. When CUR-MCT-KC was transferred to SIF, the CUR release was increased drastically between the second and the fourth incubation hour and with approximately a 100\% release after $5 \mathrm{~h}$ of incubation, as displayed in Figures 9 and 10. This can be explained with the rapid hydrolysis of MCT oils by pancreatic lipase into glycerides and free fatty acids to further release more encapsulated CUR molecules into the medium. KC belongs to a class of polysaccharides that can be degraded easily by enzymatic hydrolytic reactions, leading to the breakdown of $\alpha-1,3$ and $\beta-1,4$ glycosidic linkage and the formation of galactose and oligosaccharides. However, the pancreatic amylase cleaves $\alpha$-1,4-glycosidic linkages, but not the $\alpha-1,3$ and $\beta-1,4$-glycosidic linkages found in $\mathrm{KC}$. Therefore, $\mathrm{KC}$ is not degraded to harmful poligeenan and remained unaltered via the GIT. Nevertheless, $\alpha$-amylase has been reported to have effects on the hydrolysis of $\mathrm{KC}$ up to a certain degree, which explained the higher CUR release rate in SIF [65].

The swelling ability of CUR-MCT-KC beads were larger in $\mathrm{pH} 6.8$ compared to $\mathrm{pH} 1.2$, due to the changes in the ionic structure of $\mathrm{KC}$ when exposed to a different medium. It is believed that at $\mathrm{pH} 1.2$, the hydrogen bonds between CUR and $\mathrm{KC}$ molecules are much stronger due to the existence of a carboxylic group $(\mathrm{COOH})$ of polymers and $\mathrm{OH}$ groups which limit swelling whilst electrostatic repulsions are intensified between ionized groups (carboxylates, COO-) at $\mathrm{pH} 6.8$ of intestinal fluid, leading to greater swelling effects. Consequently, media diffusion into the beads is higher and resulting to a higher release of encapsulated CUR in SIF [59].

In SIF, the presented bile salts play a role in changing the interface, which facilitates the lipase-based degradation and thus enhances CUR release. It was reported that MCT is highly favorable over LCT, because MCT can be directly absorbed via the portal vein to the liver without the formation of chylomicrons [31]. This makes them highly preferable especially for patients with bile salt or pancreatic lipase deficiency. LCT, on the other hand, requires a more complex absorption process whereby they are modified into chylomicrons and transported via the thoracic duct lymph system to the liver for metabolism [10]. Furthermore, a shorter MCT is highly desirable due to its more rapid lipolysis to release more CUR in a short time for faster absorptions, as compared to LCT, suggesting that this couple improves the bio-accessibility of CUR to a greater extent [66]. This explains the selection of MCT as an oil phase/carrier over other types of oil in this study.

It is proposed that released CUR from hydrolyzed MCT conjugates can then diffuse through enterocytes more rapidly to interact with other lipid moieties to achieve a higher lymphatic drug transport. This is more effective than CUR-LCT conjugates. Theoretically, there are two possible mechanisms which occur to release the CUR molecules upon MCT lipolysis. MCT lipolysis releases a higher amount of CUR within a short time. Released CUR molecules attach and diffuse passively across enterocytes with a greater uptake efficiency in a shorter time due to its small size. Hydrophobic CUR molecules can then be solubilized with other fatty acids or monoglycerides into chylomicrons to be diffused into the lipid absorption pathways towards systemic blood circulation via the intestinal lymphatic transport, which bypassed the first-pass metabolism in the liver [66]. This reduces the CUR inactivation in liver and increases the amount of CUR available at cellular sites and thus enhances the drug bioavailability. Further studies will be conducted in the future to investigate the lipolysis rate and mechanism of CUR-MCT-KC gel beads.

There was no initial burst at the first hour for CUR-MCT-KC formulation. Although the burst release of CUR-MCT was higher (approximately 35\%), MCT oil is still capable of providing some shielding effect to the encapsulated CUR as compared to naked CUR molecules, which are susceptible to gastric digestion. A higher release was observed at 
the second hour from the CUR-MCT emulsion ( $>50 \%$ cumulative release) as compared to CUR-MCT-KC beads ( $<30 \%$ cumulative release), indicating the shielding effect of KC under an extreme acidic environment. Data are expressed as the mean \pm standard error of three independent experiments.

\subsubsection{In Vitro Release Profile of Beta Carotene (BC)-Loaded MCT-KC Formulation}

To achieve a comparable encapsulation and desired GIT drug release pattern, MCT-KC delivery system was also used to encapsulate another drug model, $\mathrm{BC}$ to examine the potency of MCT-KC formulation in delivering different hydrophobic molecules. A similar release trend was observed in Figure 10, when MCT-KC was employed to encapsulate BC. This indicates a constant performance of MCT-KC in delivering poorly soluble compounds via the GIT. The BC-loaded MCT-KC was revealed to be relatively stable to the gastric enzymes but can be degraded with subsequent $\mathrm{BC}$ release in the presence of pancreatin and increasing $\mathrm{pH}$ under simulated intestinal condition.

\section{Conclusions}

The ultimate use of natural polymers and food-grade composites has become an attractive approach in current pharmaceutical delivery applications, attributed to their biological origin, biodegradability, and non-toxicity. In this study, CUR was formulated into MCT-KC to improve its solubility, encapsulation, and acid resistance. FTIR spectra and SEM images have confirmed the formation of the CUR-MCT-KC formulation. MCT-KC has also demonstrated its capability to efficiently encapsulate and release CUR under the simulated upper tract GI conditions. KC was shown with improved acid resistance at extreme $\mathrm{pH} 1.2$ and simulated gastric condition, indicating its potency in offering an additional shielding effect to the encapsulated CUR. The solubility of CUR was improved using MCT two-fold with an encapsulation efficiency of $73.98 \pm 1.57 \%$ and a loading capacity of $1.32 \pm 0.03 \mathrm{mg}$ $\mathrm{CUR} / \mathrm{mL} \mathrm{MCT}$, suggesting the suitability of MCT as a carrier for hydrophobic compounds. The release profile of MCT-KC has revealed the delivery system with no premature burst of CUR and suppressed release under extreme simulated gastric condition ( $<35 \%$ release), followed by a drastic release of CUR in the mimicked intestinal condition $(\sim 100 \%$ release after $3 \mathrm{~h}$ incubation), which is favorable for further CUR absorption into the systemic circulation. CUR-MCT-KC beads can be stabled for at least one month at $-20^{\circ} \mathrm{C}$ storage conditions. On the whole, findings from this work has demonstrated CUR loaded MCTencapsulated KC formulation which could be potential materials for the development of natural, food-graded and polymer-derived delivery vehicles with enhanced characteristics for pharmaceutical applications. Further in vivo research will be carried out to explore more valuable functions and the potential of CUR-MCT-KC as an oral delivery system.

Author Contributions: Conceptualization, K.-X.T.; methodology, K.-X.T.; formal analysis, K.-X.T.; investigation, K.-X.T., L.-L.E.N.; resources, S.C.J.L.; data curation, NG, L.-L.E.N.; writing-original draft preparation, K.-X.T.; writing-review and editing, K.-X.T., S.C.J.L.; supervision, K.-X.T., S.C.J.L.; project administration, K.-X.T., L.-L.E.N.; funding acquisition, S.C.J.L. All authors have read and agreed to the published version of the manuscript.

Funding: This research was funded by the Singapore Centre for Environmental Life Sciences Engineering (SCELSE) (MOE/RCE: M4330019.C70), Ministry of Education AcRF-Tier 1 grant (RG19/18), Agri-Food and Veterinary Authority of Singapore (APF LCK102), Biomedical Research Council (BMRC)-Therapeutics Development Review (TDR-G-004-001), NTU-HSPH grant (NTU-HSPH 17002), and the Bill and Melinda Gates Foundation (OPP1199116).

Institutional Review Board Statement: Not applicable.

Informed Consent Statement: Not applicable.

Data Availability Statement: Not applicable. 
Acknowledgments: The authors wish to acknowledge the financial support from the Singapore Centre for Environmental Life Sciences Engineering (SCELSE) (MOE/RCE: M4330019.C70), Ministry of Education AcRF-Tier 1 grant (RG19/18), Agri-Food and Veterinary Authority of Singapore (APF LCK102), Biomedical Research Council (BMRC)-Therapeutics Development Review (TDRG-004-001), NTU-HSPH grant (NTU-HSPH 17002), and the Bill and Melinda Gates Foundation (OPP1199116).

Conflicts of Interest: The authors declare no conflict of interest.

\section{Appendix A}

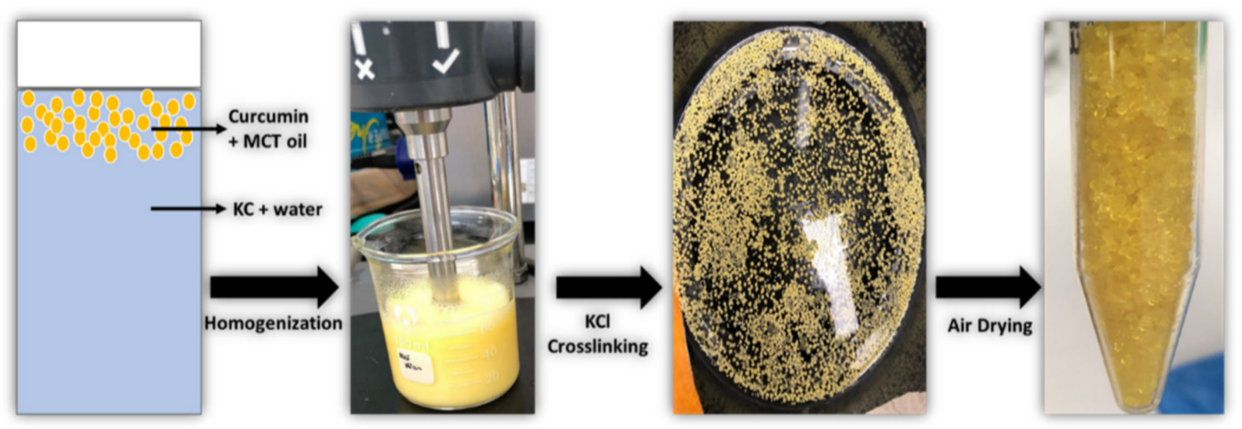

Figure A1. The preparation of CUR loaded-MCT encapsulated-KC beads.
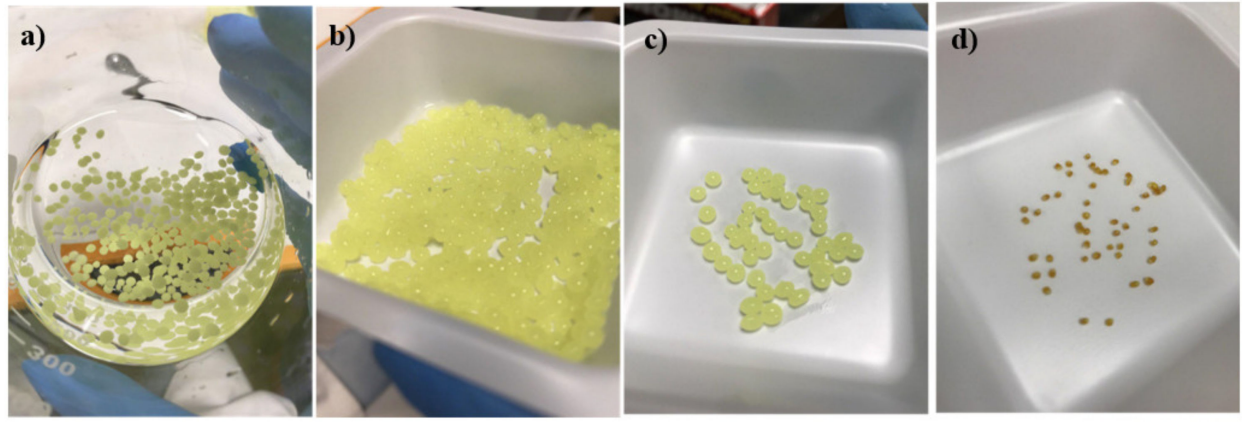

Figure A2. The $\mathrm{KCl}$ crosslinking and drying process of CUR-MCT-KC beads. (a) $5 \% \mathrm{KCl}$ was used to crosslink and solidify the CUR-MCT-KC beads, (b) the drying process of CUR-MCT-KC beads at room temperature in a light-protected container, (c) beads after 1-day of drying, (d) dried and solidified CUR-MCT-KC beads.

Table A1. The FTIR absorption bands and associated functional groups of individual CUR, MCT, and KC.

\begin{tabular}{|c|c|c|c|}
\hline Component & FTIR Absorption Band & $\begin{array}{c}\text { Descriptions-Characteristics of Different } \\
\text { Functional Group }\end{array}$ & References \\
\hline \multirow{5}{*}{ CUR } & $3504.69 \mathrm{~cm}^{-1}$ & $\begin{array}{l}\text { Assigned to the stretching vibrations of phenolic } \\
\text { hydroxyl }(\mathrm{O}-\mathrm{H}) \text { group }\end{array}$ & \multirow{5}{*}[1,47]{} \\
\hline & $1628 \mathrm{~cm}^{-1}$ & $\begin{array}{l}\text { Related to the overlapping stretching vibrations of } \\
\text { carbonyl } \mathrm{C}=\mathrm{O} \text { and alkenes } \mathrm{C}=\mathrm{C} \text { vibrations }\end{array}$ & \\
\hline & $1605 \mathrm{~cm}^{-1}$ & Indicated the stretching vibrations of benzene ring & \\
\hline & $1026 / 856 \mathrm{~cm}^{-1}$ & Attributed to the $\mathrm{C}-\mathrm{O}-\mathrm{C}$ stretching vibrations & \\
\hline & $724.24 \mathrm{~cm}^{-1}$ & Due to $\mathrm{CH}_{2}$ stretching vibrations of alkene group & \\
\hline
\end{tabular}


Table A1. Cont.

\begin{tabular}{|c|c|c|c|}
\hline Component & FTIR Absorption Band & $\begin{array}{c}\text { Descriptions-Characteristics of Different } \\
\text { Functional Group }\end{array}$ & References \\
\hline \multirow{5}{*}{ MCT } & $3472.06 \mathrm{~cm}^{-1}$ & Ascribed to the $\mathrm{C}=\mathrm{O}$ of ester & \\
\hline & $2928 \mathrm{~cm}^{-1}$ & $\begin{array}{l}\text { Due to the } \mathrm{CH}_{2} \text { and } \mathrm{CH}_{3} \text { vibration. (asymmetric } \\
\text { stretching vibrations of } \mathrm{C}-\mathrm{H} \text { of aliphatic } \mathrm{CH}_{2} \text { group; } \\
\text { asymmetric stretching vibration of } \mathrm{CH} \text { of aliphatic } \\
\mathrm{CH}_{3} \text { groups, which attributed to the alkyl of } \\
\text { triglycerides found in large amounts in vegetable oils) }\end{array}$ & \\
\hline & $1746.89 \mathrm{~cm}^{-1}$ & $\begin{array}{l}\text { Attributed to the } \mathrm{C}=\mathrm{O} \text { stretching vibration (ester } \\
\text { carbonyl functional groups of the triglycerides, } \mathrm{C}=\mathrm{O} \text { ) }\end{array}$ & \\
\hline & $1467.49 \mathrm{~cm}^{-1}$ & $\begin{array}{l}\text { Indicated the stretching of } \mathrm{CH}_{2} \text {. (bending vibration of } \\
\qquad \mathrm{C}-\mathrm{H} \text { of } \mathrm{CH}_{2} \text { and } \mathrm{CH}_{3} \text { aliphatic groups) }\end{array}$ & \\
\hline & $1159.58 \mathrm{~cm}^{-1}$ & $\begin{array}{l}\text { Assigned to } \mathrm{C}-\mathrm{O} \text { vibration (stretching vibration of } \\
\qquad \mathrm{C}-\mathrm{O} \text { ester groups) }\end{array}$ & \\
\hline \multirow{4}{*}{$\mathrm{KC}$} & $1223.64 \mathrm{~cm}^{-1}, 1243.07 \mathrm{~cm}^{-1}$ & Assigned to the $\mathrm{S}=\mathrm{O}$ of sulfate esters & \\
\hline & $1035.17 \mathrm{~cm}^{-1}, 1035.62 \mathrm{~cm}^{-1}$ & $\begin{array}{c}\text { Ascribed to the glycosidic linkage, } \mathrm{C}-\mathrm{O}-\mathrm{C} \text { of } 3,6 \\
\text { anhydro-D-galactose }\end{array}$ & \\
\hline & $921.94 \mathrm{~cm}^{-1}, 921.04 \mathrm{~cm}^{-1}$ & Due to $\mathrm{C}-\mathrm{O}$ of 3,6-anhydro-D-galactose & \\
\hline & $845.61 \mathrm{~cm}^{-1}, 844.76 \mathrm{~cm}^{-1}$ & Attributed to the $\mathrm{C}-\mathrm{O}-\mathrm{SO}_{3}$ of D-galactose-4-sulfate & \\
\hline
\end{tabular}
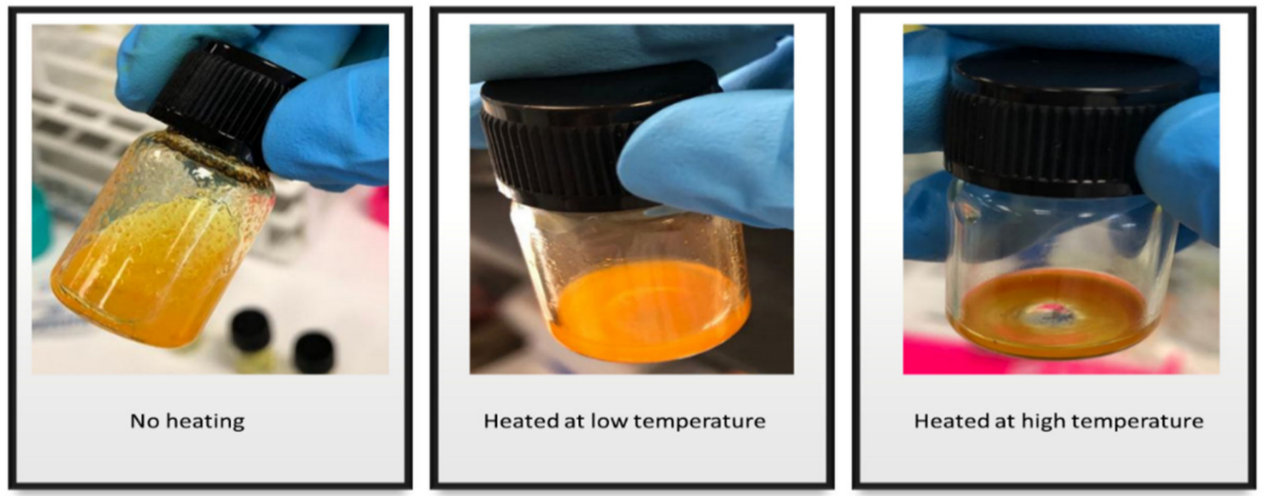

Figure A3. Effect of heat on the solubility of CUR in MCT. The CUR solubility escalates gradually with increasing temperature.

A simple experiment was also conducted to observe how CUR-MCT-KC bead behaves when incubated under acidic environment, at $37^{\circ} \mathrm{C}, \mathrm{pH} \mathrm{1.2,} \mathrm{and} 150 \mathrm{rpm}$ for $2 \mathrm{~h}$. This experiment is essential to confirm the CUR release profile and determine if KC layer capable of shielding the encapsulated CUR-MCT against gastric $\mathrm{pH}$. Based on the physical observation, CUR-MCT-KC particle was stable in acidic $\mathrm{pH}$ with reasonable swelling as seen in Figure A4. The swelling was due to hydrogel characteristics of KC, resulting in increased weight from $1.5 \mathrm{mg}$ to $2.5 \mathrm{mg}$ after 2 -h incubation at $\mathrm{pH} 1.2$. There was no yellowish color observed in the incubated medium and the amount of released CUR was determined based on the in vitro release study in both SGF and pH 1.2. 


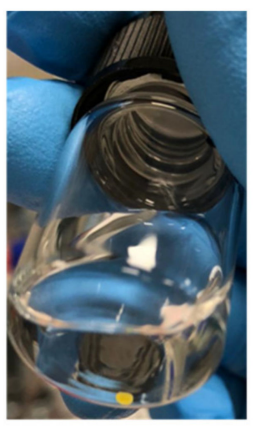

$0^{\text {th }}$ min, before incubation at $37^{\circ} \mathrm{C}$, $150 \mathrm{rpm}$

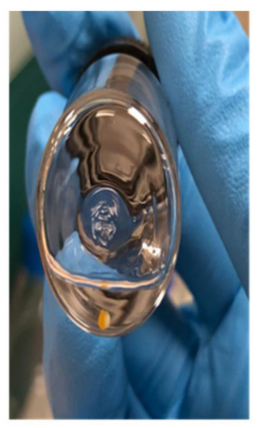

$5^{\text {th }}$ min, after incubation at $37^{\circ} \mathrm{C}$, $150 \mathrm{rpm}$

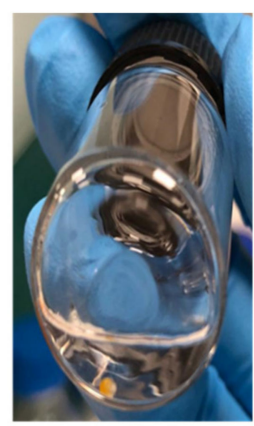

$15^{\text {th }}$ min, after incubation at $37^{\circ} \mathrm{C}$, $150 \mathrm{rpm}$

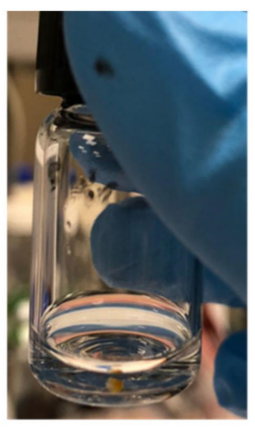

$30^{\text {th }} \mathrm{min}$, after incubation at $37^{\circ} \mathrm{C}$, $150 \mathrm{rpm}$

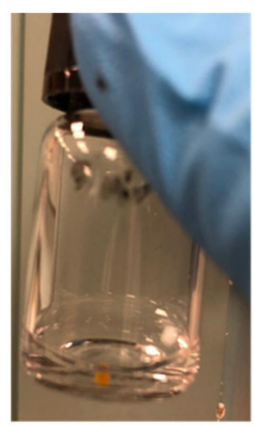

$60^{\text {th }}$ min, after incubation at $37^{\circ} \mathrm{C}$, $150 \mathrm{rpm}$

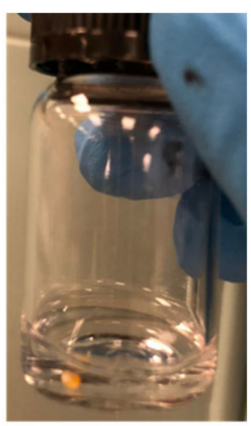

$90^{\text {th }}$ min, after incubation at $37^{\circ} \mathrm{C}$, $150 \mathrm{rpm}$

(a)

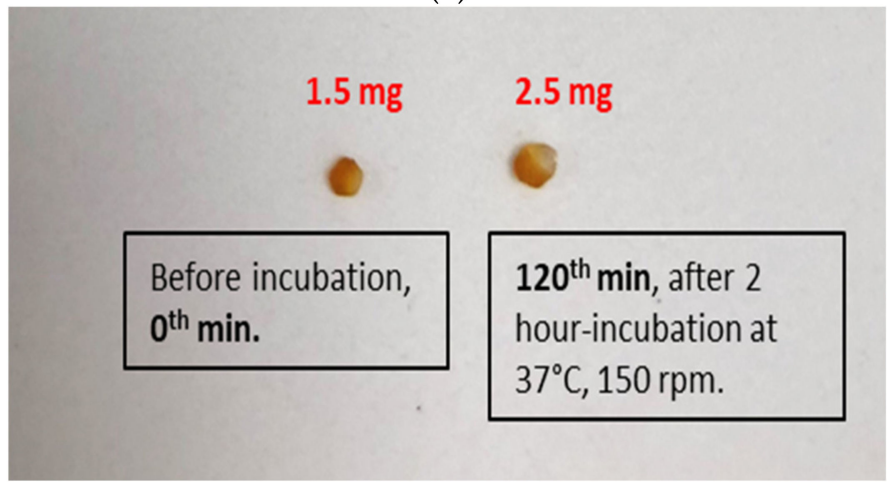

(b)

Figure A4. (a) Physical observation of CUR-MCT-KC bead at specific time intervals: 0, 5, 15, 30, 60, 90th min under the influence of acidic pH 1.2 over an incubation period of $120 \mathrm{~min}$ at $37^{\circ} \mathrm{C}$ and $150 \mathrm{rpm},(\mathbf{b})$ The different in weight of CUR-MCT-KC bead before and after the incubation $(120 \mathrm{~min})$ at $\mathrm{pH} 1.2$ at $37^{\circ} \mathrm{C}$ and $150 \mathrm{rpm}$.

\section{References}

1. Moghaddasi, F.; Housaindokht, M.R.; Darroudi, M.; Bozorgmehr, M.R.; Sadeghi, A. Synthesis of nano curcumin using black pepper oil by O/W Nanoemulsion Technique and investigation of their biological activities. LWT 2018, 92, 92-100. [CrossRef]

2. Artiga-Artigas, M.; Lanjari-Pérez, Y.; Martín-Belloso, O. Curcumin-loaded nanoemulsions stability as affected by the nature and concentration of surfactant. Food Chem. 2018, 266, 466-474. [CrossRef] [PubMed]

3. Rai, M.; Pandit, R.; Gaikwad, S.; Yadav, A.; Gade, A. Potential applications of curcumin and curcumin nanoparticles: From traditional therapeutics to modern nanomedicine. Nanotechnol. Rev. 2015, 4, 161-172. [CrossRef]

4. Zou, L.; Liu, W.; Liu, C.; Xiao, H.; McClements, D.J. Designing excipient emulsions to increase nutraceutical bioavailability: Emulsifier type influences curcumin stability and bioaccessibility by altering gastrointestinal fate. Food Funct. 2015, 6, 2475-2486. [CrossRef]

5. Kaminaga, Y.; Nagatsu, A.; Akiyama, T.; Sugimoto, N.; Yamazaki, T.; Maitani, T.; Mizukami, H. Production of unnatural glucosides of curcumin with drastically enhanced water solubility by cell suspension cultures of Catharanthus roseus. FEBS Lett. 2003, 555, 311-316. [CrossRef]

6. Pan, M.-H.; Huang, T.M.; Lin, J.K. Biotransformation of curcumin through reduction and glucuronidation in mice. Drug Metab. Dispos. 1999, 27, 486-494.

7. Lao, C.D.; Ruffin, M.T., IV; Normolle, D.; Heath, D.D.; Murray, S.I.; Bailey, J.M.; Boggs, M.E.; Crowell, J.; Rock, C.L.; Brenner, D.E. Dose escalation of a curcuminoid formulation. BMC Complement. Altern. Med. 2006, 6, 10. [CrossRef]

8. Ngan, C.L.; Basri, M.; Tripathy, M.; Karjiban, R.A.; Abdul-Malek, E. Physicochemical Characterization and Thermodynamic Studies of Nanoemulsion-Based Transdermal Delivery System for Fullerene. Sci. World J. 2014, 2014, 219035. [CrossRef]

9. Yu, H.; Shi, K.; Liu, D.; Huang, Q. Development of a food-grade organogel with high bioaccessibility and loading of curcuminoids. Food Chem. 2012, 131, 48-54. [CrossRef]

10. Traul, K.; Driedger, A.; Ingle, D.; Nakhasi, D. Review of the toxicologic properties of medium-chain triglycerides. Food Chem. Toxicol. 2000, 38, 79-98. [CrossRef]

11. Shah, N.D.; Limketkai, B.N. The Use of Medium-Chain Triglycerides in Gastrointestinal Disorders. Pract. Gastroenterol. 2017, 41, 20-28. 
12. Evingür, A.G.; Pekcan, Ö. Drying of Polyacrylamide Composite Gels Formed with Various Kappa-Carrageenan Content. J. Fluoresc. 2011, 21, 1531-1537. [CrossRef] [PubMed]

13. Das, A.K.; Sharma, M.; Mondal, D.; Prasad, K. Deep eutectic solvents as efficient solvent system for the extraction of kappacarrageenan from Kappaphycus alvarezii. Carbohydr. Polym. 2016, 136, 930-935. [CrossRef] [PubMed]

14. Sen, M.; Avci, E.N. Radiation synthesis of poly(N-vinyl-2-pyrrolidone)-kappa-carrageenan hydrogels and their use in wound dressing applications. I. Prelim. Lab. Tests. J. Biomed. Mater. Res. A 2005, 74, 187-196. [CrossRef] [PubMed]

15. Malafaya, P.B.; Silva, G.A.; Reis, R.L. Natural-origin polymers as carriers and scaffolds for biomolecules and cell delivery in tissue engineering applications. Adv. Drug Deliv. Rev. 2007, 59, 207-233. [CrossRef] [PubMed]

16. Murad, H.; Ghannam, A.; Al-Ktaifani, M.; Abbas, A.; Hawat, M. Algal sulfated carrageenan inhibits proliferation of MDA-MB-231 cells via apoptosis regulatory genes. Mol. Med. Rep. 2015, 11, 2153-2158. [CrossRef] [PubMed]

17. Mihaila, S.M.; Gaharwar, A.K.; Reis, R.L.; Marques, A.P.; Gomes, M.E.; Khademhosseini, A. Photocrosslinkable kappa-carrageenan hydrogels for tissue engineering applications. Adv. Healthc. Mater. 2013, 2, 895-907. [CrossRef]

18. World Health Organization; Joint FAO/WHO Expert Committee on Food Additives. Safety Evaluation of Certain Food Additives/Prepared by the Seventy-Ninth Meeting of the Joint FAO/WHO Expert Committee on Food Additives (JECFA); WHO Food Additives Series; World Health Organization: Geneva, Switzerland, 2015; Volume 70.

19. Scientific Committee for Food. Reports of the Scientific Committee for Food; Thirty Fifth Series; European Commission: Brussels, Belgium; Luxembourg, 1996.

20. International Agency for Research on Cancer. IARC Monographs on the Evaluation of the Carcinogenic Risk of Chemicals to Humans. Some Food Additives, Feed Additives and Naturally Occurring Substances; IARC Monographs; WHO: Geneva, Switzerland, 1983; pp. 79-94.

21. Li, L.; Ni, R.; Shao, Y.; Mao, S. Carrageenan and its applications in drug delivery. Carbohydr. Polym. 2014, 103, 1-11. [CrossRef]

22. Sathuvan, M.; Thangam, R.; Gajendiran, M.; Vivek, R.; Balasubramanian, S.; Nagaraj, S.; Gunasekaran, P.; Madhan, B.; Rengasamy, R. kappa-Carrageenan: An effective drug carrier to deliver curcumin in cancer cells and to induce apoptosis. Carbohydr. Polym. 2017, 160, 184-193. [CrossRef]

23. Joung, H.J.; Choi, M.J.; Kim, J.T.; Park, S.H.; Park, H.J.; Shin, G.H. Development of Food-Grade Curcumin Nanoemulsion and its Potential Application to Food Beverage System: Antioxidant Property and In Vitro Digestion. J. Food Sci. 2016, 81, N745-N753. [CrossRef]

24. Jiang, Y. Micro- and Nano-Encapsulation and Controlled-Release of Phenolic Compounds and Other Food Ingredients; Graduate SchoolNew Brunswick, The State University of New Jersey: New Brunswick, NJ, USA, 2009.

25. Takenaka, M.; Ohkubo, T.; Okadome, H.; Sotome, I.; Itoh, T.; Isobe, S. Effective Extraction of Curcuminoids by Grinding Turmeric (Curcuma longa) with Medium-chain Triacylglycerols. Food Sci. Technol. Res. 2013, 19, 655-659. [CrossRef]

26. Sari, T.; Mann, B.; Kumar, R.; Singh, R.; Sharma, R.; Bhardwaj, M.; Athira, S. Preparation and characterization of nanoemulsion encapsulating curcumin. Food Hydrocoll. 2015, 43, 540-546. [CrossRef]

27. Dutta, A.; Boruah, B.; Manna, A.K.; Gohain, B.; Saikia, P.M.; Dutta, R.K. Stabilization of diketo tautomer of curcumin by premicellar anionic surfactants: UV-Visible, fluorescence, tensiometric and TD-DFT evidences. Spectrochim. Acta Part A Mol. Biomol. Spectrosc. 2013, 104, 150-157. [CrossRef] [PubMed]

28. Liu, J.; Wang, H.; Wang, P.; Guo, M.; Jiang, S.; Li, X.; Jiang, S. Films based on k-carrageenan incorporated with curcumin for freshness monitoring. Food Hydrocoll. 2018, 83, 134-142. [CrossRef]

29. Soukoulis, C.; Tsevdou, M.; Andre, C.M.; Cambier, S.; Yonekura, L.; Taoukis, P.S.; Hoffmann, L. Modulation of chemical stability and in vitro bioaccessibility of beta-carotene loaded in kappa-carrageenan oil-in-gel emulsions. Food Chem. 2017, 220, 208-218. [CrossRef]

30. DeLoid, G.M.; Wang, Y.; Kapronezai, K.; Lorente, L.R.; Zhang, R.; Pyrgiotakis, G.; Konduru, N.V.; Ericsson, M.; White, J.C.; De La Torre-Roche, R.; et al. An integrated methodology for assessing the impact of food matrix and gastrointestinal effects on the biokinetics and cellular toxicity of ingested engineered nanomaterials. Part. Fibre Toxicol. 2017, 14, 40. [CrossRef]

31. Kashif, A. Encapsulation of Curcumin in O/w Nanoemulsions and Its Bioaccessibility after In Vitro Digestion; Department of Food Science, University of Massachusetts Amherst: Amherst, MA, USA, 2010.

32. Kharat, M.; Du, Z.; Zhang, G.; McClements, D.J. Physical and Chemical Stability of Curcumin in Aqueous Solutions and Emulsions: Impact of $\mathrm{pH}$, Temperature, and Molecular Environment. J. Agric. Food Chem. 2017, 65, 1525-1532. [CrossRef]

33. Ahmed, K.; Li, Y.; McClements, D.J.; Xiao, H. Nanoemulsion- and emulsion-based delivery systems for curcumin: Encapsulation and release properties. Food Chem. 2012, 132, 799-807. [CrossRef]

34. Yu, H.; Huang, Q. Improving the Oral Bioavailability of Curcumin Using Novel Organogel-Based Nanoemulsions. J. Agric. Food Chem. 2012, 60, 5373-5379. [CrossRef]

35. Zhang, Z.; Zhang, R.; Zou, L.; Chen, L.; Ahmed, Y.M.; Al Bishri, W.; Balamash, K.; McClements, D.J. Encapsulation of curcumin in polysaccharide-based hydrogel beads: Impact of bead type on lipid digestion and curcumin bioaccessibility. Food Hydrocoll. 2016, 58, 160-170. [CrossRef]

36. Richa, R.; Choudhury, A.R. Exploration of polysaccharide based nanoemulsions for stabilization and entrapment of curcumin. Int. J. Biol. Macromol. 2020, 156, 1287-1296. [CrossRef]

37. Daniel-Da-Silva, A.L.; Ferreira, L.; Gil, A.M.; Trindade, T. Synthesis and swelling behavior of temperature responsive kcarrageenan nanogels. J. Colloid Interface Sci. 2011, 355, 512-517. [CrossRef] 
38. Stone, A.K.; Nickerson, M.T. Formation and functionality of whey protein isolate-(kappa-, iota-, and lambda-type) carrageenan electrostatic complexes. Food Hydrocoll. 2012, 27, 271-277. [CrossRef]

39. Ghanam, D.; Kleinebudde, P. Suitability of $\mathrm{k}$-carrageenan pellets for the formulation of multiparticulate tablets with modified release. Int. J. Pharm. 2011, 409, 9-18. [CrossRef]

40. Bagre, A.P.; Jain, K.; Jain, N.K. Alginate coated chitosan core shell nanoparticles for oral delivery of enoxaparin: In vitro and in vivo assessment. Int. J. Pharm. 2013, 456, 31-40. [CrossRef] [PubMed]

41. Mohan, P.K.; Sreelakshmi, G.; Muraleedharan, C.; Joseph, R. Water soluble complexes of curcumin with cyclodextrins: Characterization by FT-Raman spectroscopy. Vib. Spectrosc. 2012, 62, 77-84. [CrossRef]

42. Li, J.; Lee, I.W.; Shin, G.H.; Chen, X.; Park, H.J. Curcumin-Eudragit(R) E PO solid dispersion: A simple and potent method to solve the problems of curcumin. Eur. J. Pharm. Biopharm. 2015, 94, 322-332. [CrossRef]

43. Ma, P.; Zeng, Q.; Tai, K.; He, X.; Yao, Y.; Hong, X.; Yuan, F. Preparation of curcumin-loaded emulsion using high pressure homogenization: Impact of oil phase and concentration on physicochemical stability. LWT 2017, 84, 34-46. [CrossRef]

44. Chang, C.; Wang, T.; Hu, Q.; Zhou, M.; Xue, J.; Luo, Y. Pectin coating improves physicochemical properties of caseinate/zein nanoparticles as oral delivery vehicles for curcumin. Food Hydrocoll. 2017, 70, 143-151. [CrossRef]

45. Nguyen, M.H.; Yu, H.; Kiew, T.Y.; Hadinoto, K. Cost-effective alternative to nano-encapsulation: Amorphous curcumin-chitosan nanoparticle complex exhibiting high payload and supersaturation generation. Eur. J. Pharm. Biopharm. 2015, 96, 1-10. [CrossRef]

46. Lesmes, U.; McClements, D.J. Structure-function relationships to guide rational design and fabrication of particulate food delivery systems. Trends Food Sci. Technol. 2009, 20, 448-457. [CrossRef]

47. Chen, C.; Johnston, T.D.; Jeon, H.; Gedaly, R.; McHugh, P.P.; Burke, T.G.; Ranjan, D. An in vitro study of liposomal curcumin: Stability, toxicity and biological activity in human lymphocytes and Epstein-Barr virus-transformed human B-cells. Int. J. Pharm. 2009, 366, 133-139. [CrossRef] [PubMed]

48. Madhavi, D.; Kagan, D. Bioavailability of a Sustained Release Formulation of Curcumin. Integr. Med. 2014, 13, $24-30$.

49. Kumar, A.; Ahuja, A.; Ali, J.; Baboota, S. Conundrum and therapeutic potential of curcumin in drug delivery. Crit. Rev. Ther. Drug Carr. Syst. 2010, 27, 279-312. [CrossRef] [PubMed]

50. Zsila, F.; Bikádi, Z.; Simonyi, M. Molecular basis of the Cotton effects induced by the binding of curcumin to human serum albumin. Tetrahedron Asymmetry 2003, 14, 2433-2444. [CrossRef]

51. Tønnesen, H.H.; Karlsen, J. Studies on curcumin and curcuminoids. Z. Lebensm. Unters. Forsch. 1985, 180, 402-404. [CrossRef]

52. Zhou, M.-H.; Ma, J.-S.; Li, J.; Ye, H.-R.; Huang, K.-X.; Zhao, X.-W. A k-carrageenase from a newly isolated pseudoalteromonas-like bacterium, WZUC10. Biotechnol. Bioprocess Eng. 2008, 13, 545-551. [CrossRef]

53. Khambhaty, Y.; Mody, K.; Jha, B. Purification and characterization of K-carrageenase from a novel $\gamma$-proteobacterium, Pseudomonas elongata (MTCC 5261) syn. Microbulbifer elongatus comb. Nov. Biotechnol. Bioprocess Eng. 2007, 12, 668-675. [CrossRef]

54. Rosario, N.L.; Ghaly, E.S. Matrices of water-soluble drug using natural polymer and direct compression method. Drug Dev. Ind. Pharm. 2002, 28, 975-988. [CrossRef]

55. Capron, I.; Yvon, M.; Müller, G. In-vitro gastric stability of carrageenan. Food Hydrocoll. 1996, 10, 239-244. [CrossRef]

56. Gu, S.Y.; Decker, E.A.; McClements, D.J. Influence of $\mathrm{pH}$ and iota-carrageenan concentration on physicochemical properties and stability of beta-lactoglobulin-stabilized oil-in-water emulsions. J. Agric. Food Chem. 2004, 52, 3626-3632. [CrossRef]

57. Kara, S.; Arda, E.; Kavzak, B.; Pekcan, M.Ö. Phase transitions of K-carrageenan gels in various types of salts. J. Appl. Polym. Sci. 2006, 102, 3008-3016. [CrossRef]

58. Dai, W.-G.; Dong, L.C.; Song, Y.-Q. Nanosizing of a drug/carrageenan complex to increase solubility and dissolution rate. Int. J. Pharm. 2007, 342, 201-207. [CrossRef]

59. Hezaveh, H.; Muhamad, I.I.; Noshadi, I.; Shu Fen, L.; Ngadi, N. Swelling behaviour and controlled drug release from cross-linked K-carrageenan/NaCMC hydrogel by diffusion mechanism. J. Microencapsul. 2012, 29, 368-379. [CrossRef]

60. Dafe, A.; Etemadi, H.; Zarredar, H.; Mahdavinia, G.R. Development of novel carboxymethyl cellulose/k-carrageenan blends as an enteric delivery vehicle for probiotic bacteria. Int. J. Biol. Macromol. 2017, 97, 299-307. [CrossRef]

61. Cheow, W.S.; Hadinoto, K. Biofilm-Like Lactobacillus rhamnosus Probiotics Encapsulated in Alginate and Carrageenan Microcapsules Exhibiting Enhanced Thermotolerance and Freeze-Drying Resistance. Biomacromolecules 2013, 14, 3214-3222. [CrossRef]

62. Thommes, M.; Baert, L.; Klooster, G.V.; Geldof, M.; Schueller, L.; Rosier, J.; Kleinebudde, P. Improved bioavailability of darunavir by use of $\mathrm{K}$-carrageenan versus microcrystalline cellulose as pelletisation aid. Eur. J. Pharm. Biopharm. 2009, 72, 614-620. [CrossRef]

63. Russell, T.L.; Berardi, R.R.; Barnett, J.L.; Dermentzoglou, L.C.; Jarvenpaa, K.M.; Schmaltz, S.P.; Dressman, J.B. Upper Gastrointestinal pH in Seventy-Nine Healthy, Elderly, North American Men and Women. Pharm. Res. 1993, 10, 187-196. [CrossRef]

64. McKim, J.M.; Willoughby, J.A.; Blakemore, W.R.; Weiner, M.L. Gastrointestinal Tract Digestion and Carrageenan: How Misconceptions have influenced the Understanding of Carrageenan Safety. J. Nutr. Biol. 2019, 5, 364-376.

65. Wu, S.J. Degradation of kappa-carrageenan by hydrolysis with commercial alpha-amylase. Carbohydr. Polym. 2012, 89, 394-396. [CrossRef]

66. Porter, C.J.; Trevaskis, N.L.; Charman, W.N. Lipids and lipid-based formulations: Optimizing the oral delivery of lipophilic drugs. Nat. Rev. Drug Discov. 2007, 6, 231-248. [CrossRef] 